\title{
SRNL LDRD Program Report
}

2012 
A robust Laboratory Directed Research and Development (LDRD) program is a cornerstone of the work of a National Laboratory. I am pleased that at SRNL, the LDRD program is beginning to grow and thrive, and to further enable us to demonstrate the quality of the research that is performed here every day.

The projects described in this report show the range of our capabilities, and show how we are applying those capabilities to achieve tangible results to address national needs. I think it is an excellent representation of what we do, and the value that we bring as a scientific and technical institution.

I want to thank those members of the SRNL staff, and their research partners, for their participation and the quality of their work, and I look forward to another excellent set of submittals in 2013.

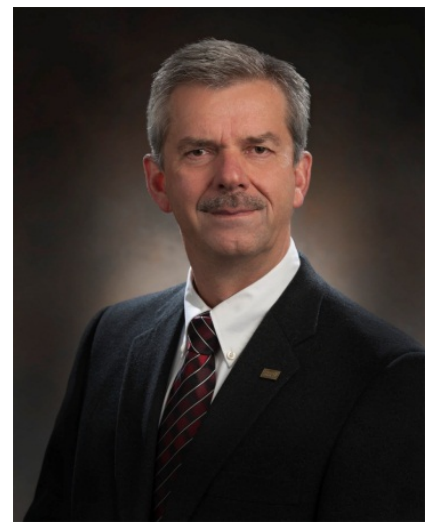

Terry Michalske

Director, SRNL 


\section{Table of Contents}

Summary of Fiscal Year 2012 Laboratory Directed Research and Development Program ......................... 1

2012 Laboratory Directed Research and Development Program Project Summaries ............................... 2

FY 2011 Strategic Initiative Project Summaries ................................................................................. 2

Reactive Gas Recycling of Used Nuclear Fuels (J. R. Gray, P. S. Korinko, D. T. Inabinett,

M. J. Martínez-Rodriguez, J. M. Becnel, R. D. Torres, B. L. García-Díaz, T. Knight, T. M. Adams).........2

Nano-Photocatalysts for Solar Fuels Applications: Conversion of $\mathrm{CO}_{2}$ to Hydrocarbons, (S. H. Murph, H. T. Sessions, Y. Zhao (UGA), K. J. Heroux, J. S. Wright, R. J. Lascola, C. E. Turick, C. E. Milliken, B. Peters, P. E. O’Rourke, B. Spencer, R. Lewis (summer intern), C. R. Shick, A. Greytak (USC), S.

Retterer (ORNL), J. Hudson (Clemson University)) ........................................................................ 4

Risk Reduction and Process Optimization for Engineered Algae Production Systems

(M. A. Heitkamp ${ }^{1}$, C. E. Bagwell ${ }^{1}$, M. Piskorski ${ }^{2}$, R. Barnwell ${ }^{3}$, A. Abernathy ${ }^{3}$, P. Moeller ${ }^{4}$,

P. Zimba $)^{5}{ }^{1}$ SRNL, ${ }^{2}$ USCA, ${ }^{3}$ SRNL Intern, ${ }^{4}$ NOAA, ${ }^{5}$ Texas A\&M) ................................................ 6

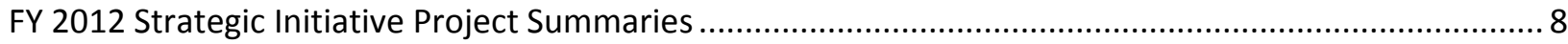

Non-Aqueous Electrochemical Fluorination of Used Nuclear Fuel as an Advanced Separation

Process (B. L. Garcia-Diaz, L. C. Olson, M. J. Martinez-Rodriguez, D. R. Click, J. R. Gray) .................... 8

Characterization of Chemical Changes Observed for Uranium and Plutonium Particulate Materials

(N. J. Bridges, G. A. Fugate, M. R. Kriz, J. J. Pittman, M. J. Siegfried, M. S. Wellons) ......................... 10

Unveiling micro-to-nano fluidics physico-chemical phenomena for advance separation and ultrasensitive detection of nuclear species (P-S. Lam, M. G. Bronikowski, A. E. Méndez-Torres) .............. 13

LDRD-2012-30 Hardware in the Loop Electrical Grid Simulator (J. V. Cordaro, G. H. Fisher Jr.,

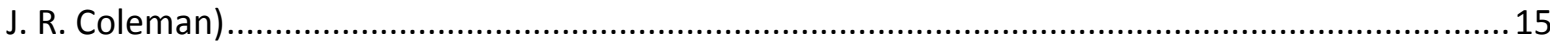

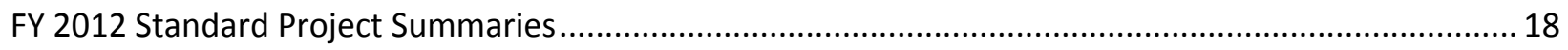

Tunable Hydrophobic/philic Reaction Sites on Multifunctional Catalysts for the Redox of Lithium for Improved Energy Storage (E. B. Fox (PI), R. E. Fuentes, S. Kendrick, H. R. Colón-Mercado) ............... 18

Redox Catalyst Development for Improving Electrolytic Cells (R. E. Fuentes, H. R. Colón-Mercado,

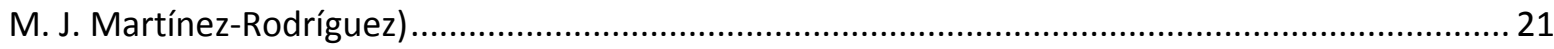

Implementation and Testing Organic Thin Film Systems as Beta Radiation Sensors (A. L. Washington

II, L. C. Teague, J. R. Coleman, R. J. Lascola (SRNL); J. Anthony (University of Kentucky)) ................23

New Materials for Hybrid Lithium-Ion Battery (J.A. Teprovich Jr., H.R. Colón-Mercado, S. Greenway, L. Teague, R. Zidan) 26

Novel Materials for Actinide Separations (K. M. L. Taylor-Pashow (SRNL); W. Lin (UNC); C. W. Abney (UNC); M. Carboni (UNC)) 28 
Removal of Aqueous ${ }^{99} \mathrm{Tc},{ }^{129} \mathrm{I}$, and ${ }^{137} \mathrm{Cs}$ with Reactive, Low-Cost Materials (D. I. Kaplan, A. S. Knox,

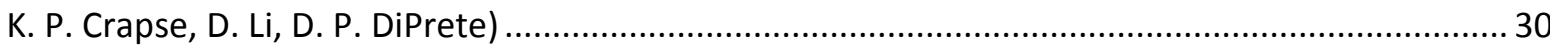

Evaluation of Hydrogen Isotope Exchange Methodology on Adsorbents for Tritium Removal (G. A.

Morgan, Jr.) .

Application of Cooling Towers for the Collection, Concentration, and Detection of Biological Agents, Radionuclides, and Elemental Chemicals (R. L. Brigmon, D. I. Kaplan, W. L. Jones, M. T. Kingsley (Savannah River National Laboratory, Aiken, SC); S. Leskinen, E. Kearns, D. Lim, (University of South

Florida (USF), Tampa))

Smart Nanophase Extractors for Environmental Remediation and Liquid Waste Clean-up

(L. T. Sexton, R. D. Torres, S. M. Serkiz, B. L. Brown) 35

FY 2012 Quick Hit Summaries

Alignment of Nanorods into Coherent Structures Using Evanescent Electric Fields (R. J. Lascola, P. E. O'Rourke, S. H. Murph, J. S. Wright, K. J. Heroux, B. Peters) ...................................................... 37

Novel Concepts for Isotopic Separation of ${ }^{3} \mathrm{He} /{ }^{4} \mathrm{He}$ (L. E. Roy (PI), H. L. Watson, H. L. Nigg).............. 39

Application of Quantum Cascade Lasers to IR Spectroscopy (T. L. White, P. E. O'Rourke, W. A. Spencer, F. Fondeur)

GrayQb - A 3D Radiation Mapping Device (E. B. Farfan and R. J. Coleman) 


\section{Summary of Fiscal Year 2012 Laboratory Directed Research and Development Program}

The Laboratory Directed Research and Development (LDRD) Program at Savannah River National Laboratory (SRNL) supports high quality research and development to further the mission of the Department of Energy. Research teams supported through LDRD in FY12 yielded quality research in a variety of technical focus areas. Tangible accomplishments resulted from persistent efforts in the following areas:

External Funding

Over $\$ 6 \mathrm{M}$ new project funds were awarded this past year as a direct result of efforts related to FY12 LDRD projects. External program funds related to FY12 LDRD funded projects have been obtained from the following agencies:

- EERE (Office of Biomass, Sunshot Initiative, Office of Vehicle Technology)

- NNSA

- DOE-NE (University Programs)

Scientific Productivity

Intellectual Property: Nine invention disclosures were submitted related to research supported through the LDRD program. Two patent applications were filed in FY12, including:

- Nanocatalysts and Shape Selective Method for Production of Nanocatalysts

- Water Cooling towers and Other Man-made Aquatic Systems as Environmental collection Systems for Agents of Concern

Publications: Four peer-reviewed manuscripts and one book chapter were published in FY12.

Post-Doctoral and Student Involvement

- Twelve post-doctoral researchers were members of 13 LDRD supported research teams in FY12, six performed research at SRNL.

- Eight LDRD projects supported graduate student involvement in FY12. Four projects supported undergraduate participation. 


\title{
2012 Laboratory Directed Research and Development Program Project Summaries
}

\section{FY 2011 Strategic Initiative Project Summaries}

\author{
Reactive Gas Recycling of Used Nuclear Fuels (J. R. Gray, P. S. Korinko, D. T. Inabinett, \\ M. J. Martínez-Rodriguez, J. M. Becnel, R. D. Torres, B. L. García-Díaz, T. Knight, T. M. Adams)
}

Abstract: Nuclear Energy will be a key element in the development of a long long-term, clean sustainable energy solution in the Unites States. Development of advanced, economical Used Nuclear Fuel (UNF) recycling technologies to close the fuel cycle is an important facet of this solution. Widespread implementation of commercial UNF recycling necessitates the development of low-cost, safe, reduced-footprint technologies that are inherently proliferation-resistant. SRNL has been developing novel dry reactive gas recycling $(R G R)$ technologies which could be integrated with multiple fuel cycle options and reactor from designs. Current SRNL research efforts indicate the possibility of developing both fully closed and limited recycling schemes based on dry process operations. The reported research is comprised of theoretical analyses and experimental characterization to advance the development of novel dry recycling concepts for UNF. This program has developed the preliminary scientific basis for candidate technologies that could allow us to develop a tailored separations process for low-cost fuel recycling, liquid waste reduction, and proliferation resistance.

The research reported that the overarching objective of moving SRNL towards a technical and business leadership position in the development of alternate UNF recycle processes. SRNL is proposing to simultaneously react UNF materials with a mixed-gas environment as a partial alternative to current aqueous technologies. This research would leverage SRNL leadership in aqueous processing and adapt it to investigate emerging dry recycle technologies. This dry process can be designed to efficiently help remove the zircaloy cladding from the UNF rods and subsequently separate the actinides in proliferation-resistant schemes while at the same time making these valuable resources available for recycle/reuse in commercial or advanced burner reactor fuels. The development of dry separations and recycle technologies was seen as a transformational technology for the advancement of next generation nuclear energy production. The reported work consists of two main thrusts which extend to particular approaches of RGR technology development - theoretical analysis and experimental data collection. The first aspect of the research consisted of theoretical calculation tools comprising thermodynamic prediction and reaction pathway and kinetic analysis. The second key area involved the use of experimental studies to test hypotheses and process conditions for the targeted approaches to dry UNF recycle. Using these two aspects of the project has allowed the development of a flowsheet model that can be used to assist in developing a framework for dry UNF recycle technologies at SRNL. Significant progress has been accomplished in each of the areas investigated for the development of the RGR technologies for UNF. Two sections of a theoretical process flow sheet were selected for development in this project - these were chemical decladding of zircaloy fuel rods to accentuate or potentially replace 
mechanical decladding, and the second was the investigation of alternative solid fluorinators for UNF treatment.

The first area seeks to leverage SRNL's technical leadership in the exposure of hydrogen and its isotopes to a variety of metal substrates including zirconium. Hydrogen decrepitation experiments were conducted on Zircoloy alloys that were in the as-received condition, surface ground, and oxidized to simulate 1500 days in reactor, roughly three refueling cycles. The manifold apparatus that was used for these experiments has sufficient flexibility so a broad range of hydrogen to zirconium ratios can be accommodated. The testing for these experiments used an $\mathrm{H} / \mathrm{Zr}$ of 2.0.

Previous efforts demonstrated that decrepitation could be achieved with Zircoloy clean or as-received condition. Thus experiments were conducted with modestly oxidized samples. These results from ostensibly clean and intentionally oxidized Zircoloy are shown in Figure 1. The oxidized samples have indeed decrepitated, although not to the same extent as the clean samples. While there is need for additional development and scale up, the methodology is proven to be effective and could be used to obviate mechanical decladding, and at minimum it will certainly enhance current mechanical decladding processes.

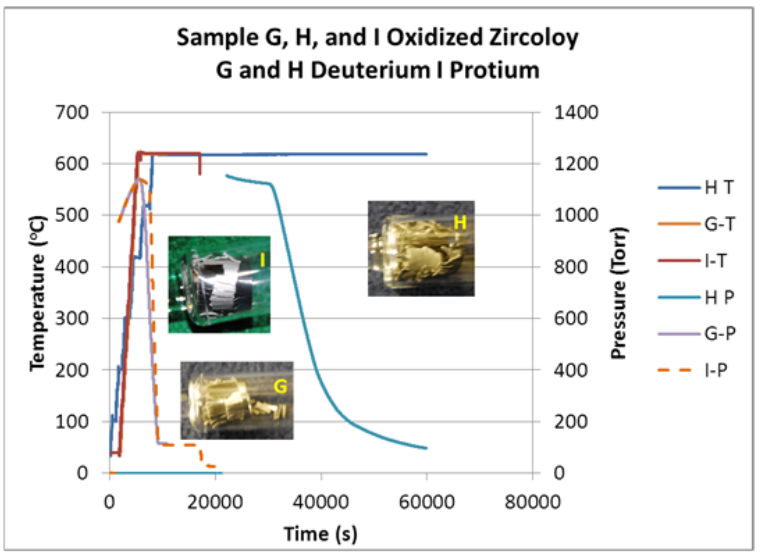

Samples B, D, E and F

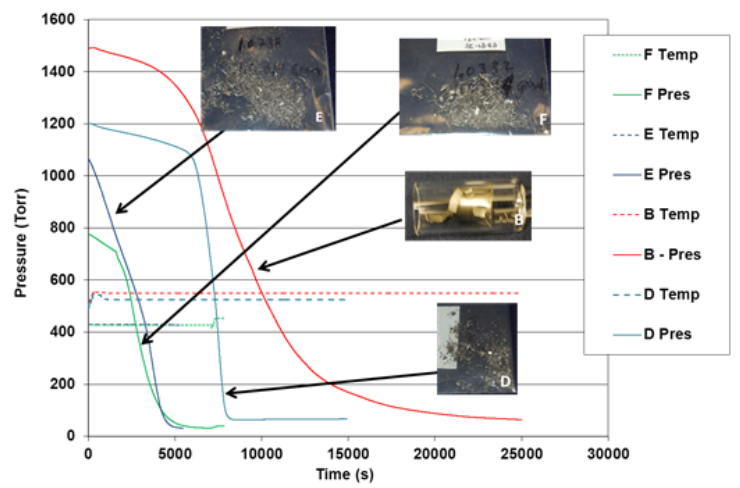

Figure 1. Hydride Decreptiation of Zircaloy Rods
Preliminary calculations were conducted which point to the potential efficacy of using $\mathrm{XeF}_{2}$ and $\mathrm{NH}_{4} \mathrm{HF}_{2}$ as alternate fluorinating agents for separation of key UNF species. Both of these materials have been examined in the literature and are known to have some reactivity with uranium oxide compounds, but have not been extensively tested against other materials present in a theoretical UNF matrix. Additionally, due to the fact that these materials are present in the solid phase at room temperature, they allow for compact storage of the $F_{2}$ molecules as compared to the same amount of $F_{2}$ stored in the gas phase. Thermal gravimetric and differential thermal analysis techniques were used to determine to what extent these solid fluorinators might fluorinate both clean surrogate materials representative of both volatile and nonvolatile fluoride species, as well as uranium oxide materials. Thus far it appears that the clean surrogate materials are fluorinating as expected to form such products as $\mathrm{SrF}_{2}$ and $\mathrm{MoF}_{6}$, however experiments conducted with both $\mathrm{XeF}_{2}$ and $\mathrm{NH}_{4} \mathrm{HF}_{2}$ have not yielded evidence of fluorination of $\mathrm{UO}_{2}$ or $\mathrm{U}_{3} \mathrm{O}_{8}$ to form either $\mathrm{UF}_{4}$ or $\mathrm{UF}_{6}$. This area of study is being continued in the laboratory of Prof. Knight by graduate student Dillon Inabinett who will be studying alternate fluorination species as part of his Master's degree thesis. Dillon will be applying both experimental techniques as well as advanced 
thermodynamic calculations such as FactSage and ASPEN $^{+}$software packages to extend the preliminary calculations that have already been conducted in the current project.

This project has successfully demonstrated a chemical decladding technology that could be used to potentially replace or accentuate currently used mechanical decladding technologies by decrepitating the zircaloy material in controlled particle sizes ranging from fine powders to chunks. Furthermore, a study has been initiated investigating the use of alternate solid-phase fluorinators to serve as potential separations reagents for volatility separations processes. These experimental data are sutured with theoretical calculations and captured in process scale flow diagrams. These results will be used to actively pursue future funding while integrating with other separations technologies being investigated at SRNL such as fluoride-based molten salt pyroprocessing.

\section{Nano-Photocatalysts for Solar Fuels Applications: Conversion of $\mathrm{CO}_{2}$ to Hydrocarbons, (S. H. Murph, H. T. Sessions, Y. Zhao (UGA), K. J. Heroux, J. S. Wright, R. J. Lascola, C. E. Turick, C. E. Milliken, B. Peters, P. E. O’Rourke, B. Spencer, R. Lewis (summer intern), C. R. Shick, A. Greytak (USC), S. Retterer (ORNL), J. Hudson (Clemson University))}

Abstract: A multi-interdisciplinary team of researchers from SRNL, UGA, USC, ORNL, and Clemson University were able to demonstrate for the first time solar conversion of carbon dioxide and water vapor to methane, methanol, carbon monoxide, hydrogen and potentially other chemicals by exposure of titania-based nanophotocatlysts under ambient light only. The photocatalytic performance of the nanoarchitectures developed here was highly dependent upon nanoparticle's shape, morphology, composition, crystallinity, solar activity, and illumination. Bottom up and top down approaches were the main key concepts that allowed production of tunable nanohybrid ordered-arrays of titania-based catalysts with multifunctional, broadband electromagnetic response and precisely defined properties and functions. A variety of state-of-the-art optical, surface, physical and chemical techniques were used to characterize the library of the active nanophotocatalysts. The novel multifunctional nanomaterials developed here were also efficient for monitoring and photodegradation of organic contaminants potentially present in the environment. They were also excellent substrates for chemical and biological detection providing molecular structural information together with ultrasensitive detection limits via SERS and/or fluorescence spectroscopy and potential candidates for tritium related processes and other energy related applications (fuel cells).

The development of efficient nano-photocatalysts for renewable fuel production from environmental carbon dioxide $\left(\mathrm{CO}_{2}\right)$ and water precursorshas the potential to enable long term, sustainable production of storable, transportable fuels. While this conversion can be achieved with the help of photocatalysts, the optical to chemical conversion efficiency has generally been very low, at less than $0.5 \%$. This is because most of photocatalysts have a very narrow band edge absorbance, and provide limited amounts of free electrons and protons on the surface for catalytic reactions. The overall goal of this proposal was the creation of actively controllable and tunable nano-photocatalysts with multifunctional, broadband electromagnetic response for solar energy conversion of $\mathrm{CO}_{2}$ to fuel. Two interconnected directions of research were explored to enhance photocatalytic carbon dioxide conversion rates: (a) Development of innovative and tunable nanohybrid architectures with different topography, crystal 
structure, relative composition and interfacial area in a controllable manner for efficiently coupling lightharvesting, photo-redox processes, and photocatalytic behaviors; (b) Testing and evaluation of nanophotocatalytic system response toward conversion of $\mathrm{CO}_{2}$ to fuel.

Titania has been considered the most appropriate candidate for photocatalytic processes due to its powerful oxidation capability, superior charge transport, and corrosion resistance. Additionally, $\mathrm{TiO}_{2}$ is inexpensive, has reasonable activity, and is abundant. The absorption of photons from natural light in photocatalytic materials generates electrons and holes which could drive energy conversion processes in the solar-driven photoreduction of $\mathrm{CO}_{2}$ and/or water-splitting for hydrogen fuels. Despite these attributes, the efficiency of $\mathrm{TiO}_{2}$ for photovoltaic and photocatalytic applications is severely limited by its large band gap ( $3.2 \mathrm{eV}$ ) and rapid charge carrier recombination dynamics which means that $\mathrm{TiO}_{2}$ can use less than approximately $5 \%$ of the solar spectrum. Efforts have been devoted to producing and sensitizing $\mathrm{TiO}_{2}$ nanomaterials with nanoparticles that absorb light at lower photon energies, thus extending absorption into the visible light region of the spectrum. Coupling of pure $\mathrm{TiO}_{2}$ with noble metals (NPs), quantum dots (QDs) and other oxides extends the adsorption of solar energy in visible region and generates more electron-hole pairs, due to the smaller band gap of those sensitizers. This results in higher photocatalytic efficiency for the $\mathrm{CO}_{2}$ conversion process. Photocatalytic conversion of $\mathrm{CO}_{2}$ was monitored with either an in-house designed or a purchased solar-gas chambers. Both systems were equipped with gas inlet and outlet, optical window, sample stage holder, heater and temperature sensor that allowed evaluation and optimization of catalysts for solar fuels applications. The type and amount of hydrocarbons and other gases generated through prolonged light-soaking was quantified by both residual gas analysis and a gas-chromatography.

A library of inexpensive, active and tunable one-dimensional titania-based nanohybrid architectures catalysts with multifunctional, and tunable broadband electromagnetic response was amassed by a combination of solution chemistries, glancing angle deposition (GLAD) and nanolithography. Light absorption for these materials was tuned either by surface decorations of metal nano-island, doping, core-shell design, or a combination of multi-badgap nanocatalysts. Specifically, we engineered the bandgap of the $\mathrm{TiO}_{2}$ photocatalytic materials with a variety of sizes, shapes and morphologies of NPs ( $\mathrm{Au}, \mathrm{Ag}, \mathrm{Pt}, \mathrm{Au}-\mathrm{Cu}), \mathrm{QDs}\left(\mathrm{CdS}, \mathrm{CdSe}, \mathrm{Cd}_{x} \mathrm{Zn}_{1-x} \mathrm{~S}\right)$, and other oxide $\left(\mathrm{SiO}_{2}, \mathrm{WO}_{3}\right.$ and $\left.\mathrm{Fe}_{2} \mathrm{O}_{3}\right)$ sensitizers to maximize the reaction channels for the photocatalytic conversion. A variety of techniques were assayed for characterization and optimization of the novel nanomaterials produced including SEM, HRTEM, UVVIS, ellipsometry, fluorescence, XRD, EDX, AFM, QCM, Raman, $\xi$ potential, ICP-MS, and reflectance spectroscopy among others. Mathematical and theoretical calculations (e.g DFT, Boltzmann equation, Tauc plots, Lambert-Beer's law) were also used to validate ongoing experimental work. The photocatalytic performance of the newly developed catalysts was also explored. There are several major findings from our studies. Annealing $\mathrm{TiO}_{2}$ at temperatures above $300{ }^{\circ} \mathrm{C}$ increased their photodegradation activity toward model organic analytes. During the annealing process, the crystal structure changes from amorphous to anatase, and the number of defect sites is reduced. Crystal structure thus improves the photocatalytic efficiency as follows: anatase>rutile>brookite. Higher surface area provided by core-shell oxide layers also improves photocatalytic activity. For example, $\mathrm{SiO}_{2}-\mathrm{TiO}_{2}$-coated post arrays exhibit faster degradation kinetics than the $\mathrm{TiO}_{2}$ coated arrays $(\sim 72 \%$ 
improvement); similar results were demonstrated with $\mathrm{Fe}_{2} \mathrm{O}_{3}-\mathrm{TiO}_{2}$ and $\mathrm{TiO}_{2}-\mathrm{WO}_{3}$. The $\mathrm{TiO}_{2}$ layer may adhere better to $\mathrm{SiO}_{2}$ rather than $\mathrm{Si}$ due to the higher porosity of the $\mathrm{SiO}_{2}$ surface. A shape dependence response was also discovered: diamond>squares>triangles>spheres. Nanoparticles with more edges and corners were found to be more reactive than spherical particles due to different crystallographic orientation of $\mathrm{TiO}_{2}$. Nanophotocatalysts have been evaluated for photo catalytic reduction of $\mathrm{CO}_{2}$ with $\mathrm{H}_{2} \mathrm{O}$ vapor by exposing three $1 \mathrm{~cm}^{2}$ of catalyst in $\sim 20 \mathrm{cc}$ gas mixture and under ambient light, UV and/or UV-Vis illumination. Amongst the $\mathrm{TiO}_{2}$-based nanocatalysts investigated, $\mathrm{Au}-\mathrm{Cu}-\mathrm{TiO}_{2} \mathrm{WO}_{3}-\mathrm{TiO}_{2}$, and $\mathrm{Pt}$ hollow- $\mathrm{Cd}_{x} \mathrm{Zn}_{1-x} \mathrm{~S}-\mathrm{TiO}_{2}$ nanocatalyst showed maximum activity in terms of $\mathrm{CH}_{3} \mathrm{OH}, \mathrm{CH}_{4}, \mathrm{CO}$ and $\mathrm{H}_{2}$ and formation (hundreds ppm). In the presence of a hole scavenger (e.g. organic molecules) the photogenerated holes were effectively consumed at a rate that exceeded recombination. This resulted in a significant increase (3-5 times) in gas production. The co-generation of $\mathrm{CO}$ and $\mathrm{H}_{2}$ from waste chemicals by solar energy and can also drive $\mathrm{CO}_{2}$ fixation in engineered anaerobic, acetogenic bacteria to synthesize high energy-density liquid fuels. We exposed anaerobic bacteria in a CO environment and we produced acetate. Major factors influencing activity and product selectivity include: choice of catalyst preparation methods, crystal structure, and particle size; sensitizer composition; presence of hole scavenger; band energy characteristics of the catalyst and the wavelength of the radiation.

A technology was developed that shows promise toward reduction of the levels of $\mathrm{CO}_{2}$ in the atmosphere and at the same time captures a renewable source of energy (solar radiation), photocatalytically transforming solar energy into a form that is more readily stored, and used, thus preserving the large investments made on fossil fuels.

\section{Risk Reduction and Process Optimization for Engineered Algae Production Systems

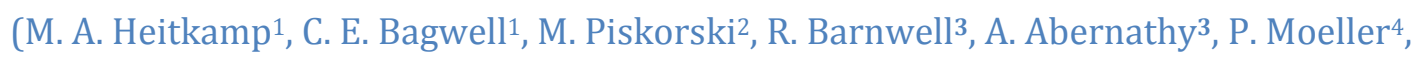 P. Zimba5)1SRNL, 2USCA, ${ }^{3}$ SRNL Intern, ${ }^{4}$ NOAA, ${ }^{5}$ Texas A\&M)}

Abstract: This research investigation focused on microscopic algae as a potential new source of clean burning liquid fuels. At the cellular level, solar energy is captured to power the conversion of carbon dioxide into liquid fuels. The economic viability and sustainability of large scale production of algae is, in part, hinged on scientific advances that can increase growth rates and biofuel yields. Open pond systems are the only economically viable option for fuel production from algae; however, the challenges include microbial contamination, consistency of yields, fuel quality, and re-use of algal by-products. The goals of this research were to leverage fundamental science for solutions that will overcome these challenges by ensuring the safety, and contributing meaningfully to the sustainability and cost competitiveness of microalgae production systems.

The specific project objectives were aligned with information collected from industry leaders, the research community, and the future direction of the DOE EERE Biomass Program and other federal agencies to provide the best fit and highest growth potential for SRNL in the rapidly expanding green bio-energy market. Large scale production of algae is a resource intensive process; thus, there is tremendous need for smart systems that are flexible in their utilization of various waste streams for the growth of algae and conversion of $\mathrm{CO}_{2}$ into high value products. We focused on the following gaps in 
the field; 1) improvements in algae growth and production efficiency, 2) sustainability and explanation for observed variability in large production systems, and 3) production of novel metabolites.

Biofuel candidate strains of algae were grown under a suite of conditions intended to mimic discrete stages or scenarios in real-world production of algal biomass, co-product induction, or biofuel substrate accumulation. Algal biomass and production waters were systematically analyzed, permitting an integrated meta-analysis to establish connectivity networks between algal physiology, bacterial interactions, cytotoxicity triggers, nutrient utilization, inorganic contaminant fate and speciation, VOCs, and hydrocarbon responsiveness.

Micro-algae do not exist in isolation; they are constantly surrounded by and interacting with a bacterial flora and the interspecies communication that occurs is critically important for sustainable management of algae for biofuels production. Attempts to produce bacterial-free algae cultures mostly failed; 3 axenic cultures were produced however the host alga died within 2 generations. Our work shows that biofuel candidate strains of algae are dependent on bacterial symbionts for maintenance, growth, and development; therefore the bacterial component of microalgae cultures must be understood and equally cultivated to achieve sustainable biofuels production.

Nearly 300 algae samples from freshwater wetlands and outdoor production raceways were surveyed for the co-occurrence of microbes capable of producing growth promoting phytohormones, chiefly indole auxins. Nearly $60 \%$ of the samples reacted positively for tryptophan-dependent auxin production. This high frequency of association is remarkable and may imply the selective maintenance of this functional attribute or of a core microbiome by diverse green microalgae. Auxin producing cultures were serially diluted and streak plated to bacterial isolation. Approximately 150 pure culture strains were confirmed for auxin producing activity and identified by partial 16S rRNA gene sequencing. Strains belonged to several proteobacterial classes (alpha, beta, gamma), as well as both Gram (+) phyla, Actinobacteria and Firmicutes. Several strains were closely related to authentic plant growth promoting bacteria (PGPB) though the majority did align with taxa having a multitude of beneficial interactions with terrestrial and aquatic plants. The importance of interspecies interactions for algae biofuels cannot be disregarded; the ability to purposefully design and engineer a microbiome for biofuel algae would mark a truly revolutionary advancement in the biofuels industry.

Systematic studies revealed that exposure to discrete concentrations of indole-3-acetic acid promotes the production and cellular accumulation of photopigments necessary for the capture and conversion of solar energy into chemical energy. A typical algal culture is asynchronous, meaning that all of the cells within the culture exist in various states of activity, development, and death. Conversely, treatment with auxin can be used to consistently and effectively synchronize mass algal cultures at the cellular / metabolic levels; total cell volume shrinks (allowing cells to pack more closely in dense culture) and the photosynthetic apparatus increases to fill the entire cellular volume. This finding is the basis for a novel bioprocess under development whereby growers can 'prime' algae cultures in order to maximize photosynthetic conversion into cellular biomass, biofuel substrate, or high value co-products. 
Reciprocal experiments have confirmed a linkage between iron bioavailability and the production of cytotoxic metabolites by biofuel candidate strains of algae. Green microalgae have never been shown to produce toxins, our work is the first. Moreover, we have successfully and clearly demonstrated this outcome to be reproducible, predictable, and on/off production of at least 6 different toxin compound families is directly linked to iron. This work has direct implications for production of value added coproducts and the use of algal biomass in foods production. Beyond consideration of risk, toxins are now receiving attention by industry leaders as a natural means of controlling unwanted contamination in open pond systems though the molecular 'switch' needs to be identified. Secondly, there are unlimited applications and markets for the discovery of novel compounds with therapeutic properties.

Microalgae can be part of the solution to our nations need for secure, sustainable, and clean energy. The process is unquestionably feasible; now scientific breakthroughs are needed to make algae-based biofuels cost competitive with petroleum fuels. This can only be achieved by realizing the full potential of algae as a valued commodity and expanding the algae-fuel life cycle accordingly. This project has made major strides by adapting to the emerging needs of this rapidly evolving field. In a very short time we have been able to leverage fundamental science to make important new discoveries which have transitioned into the conceptualization of potentially game changing biotechnologies for the algae biofuels industry.

\section{FY 2012 Strategic Initiative Project Summaries}

\section{Non-Aqueous Electrochemical Fluorination of Used Nuclear Fuel as an Advanced Separation Process (B. L. Garcia-Diaz, L. C. Olson, M. J. Martinez-Rodriguez, D. R. Click, J. R. Gray)}

Abstract: The development of efficient and environmentally benign methods to reprocess used nuclear fuel (UNF) will be enabling technologies for the nuclear renaissance. Non-aqueous methods of fuel processing are currently being developed to reprocess UNF with significantly reduced waste volumes. An electrochemical fluorination process is being developed that is capable of efficiently separating gas phase uranium through potential control of the reaction. Thermodynamic modeling has shown that a galvanic reaction between UNF and a fluorinating agent such as $\mathrm{NF}_{3}$ in a molten fluoride electrolyte is possible and could selectively fluorinate $U$ to $U F_{6}$. Reactor and separation systems have been developed to prove this concept experimentally.

The use of electrochemical and fluorination methods for reprocessing UNF and been proposed and researched by various groups, but combinations of electrochemical and fluorination methods have not been investigated. Fluorinating agents are extremely potent oxidizing agents and should be able to electrochemically fluorinate metallic UNF with no input of electricity. The technical object of this project is to demonstrate the thermodynamic feasibility of electrochemical fluorination for UNF separations, demonstrate the electrochemical fluorination process, and develop flowsheets to show how this technique can be incorporated into state-of-the-art reprocessing schemes. 
Thermodynamic calculations of half-cell potentials for potential electrochemical reactions for all UNF constituents were performed using HSC Chemistry. The analysis normalized the potentials against a $\mathrm{Ni} / \mathrm{NiF}_{2}$ reference that is used for many molten fluoride electrochemical cells. Thermodynamic analysis was conducted at temperatures between $0^{\circ} \mathrm{C}$ and $800^{\circ} \mathrm{C}$. Operation is anticipated to be best in the range of $500-600^{\circ} \mathrm{C}$ with a eutectic mixture of LiF-NaFKF (FLiNaK) to keep corrosion reactions to a minimum. The electrochemical reactor consists of a glassy carbon crucible to hold the molten salt and glassy carbon porous cathode for $\mathrm{NF}_{3}$ reduction. An engineering drawing for the reactor is shown in Figure 1. Depleted uranium (DU) turnings are used as surrogate material for UNF to prove the evolution of

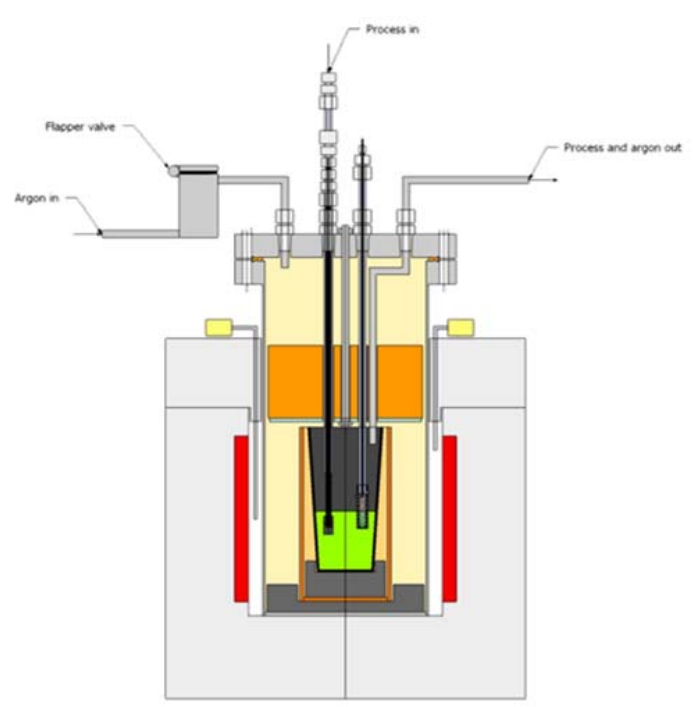

Figure 1. Reactor Design for Electrochemical Fluorination $\mathrm{UF}_{6}$. Thermodynamic calculations have also been performed to demonstrate the feasibility of reduction of oxide UNF fuel to its metallic state using HSC chemistry.

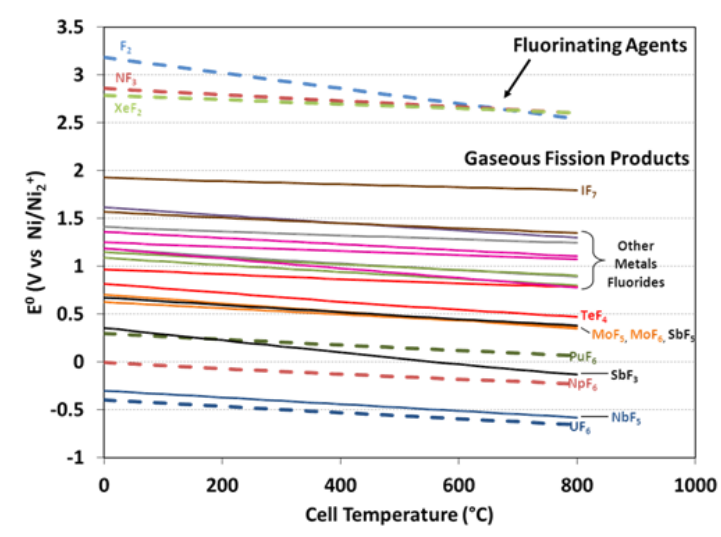

Figure 2. Equilibrium potentials for gaseous fluoride fission products.
Electrochemical modeling of the electrode reactions for the electrochemical fluorination reactions and the oxide reduction reactions was highly successful. A complete analysis of the electrochemical potentials was performed that showed that $\mathrm{UF}_{6}$ would be the first gaseous fission product to be evolved from metallic UNF and would therefore facilitate separation from other UNF components. Figure 2 shows the equilibrium potentials for the gaseous products and common fluorinating agents. The results in Figure 2 show that fluorination of $U$ to $U_{6}$ has the lowest equilibrium potential of any electrochemical potential that produces a gaseous fission product. All of the

fluorinating agents also have very high equilibrium potentials that indicate that can easily be reduced electrochemically. The kinetics of the anode and cathode reactions should not be a significant problem even when trying to selectively oxidize UNF components given the $>300-\mathrm{mV}$ gap between gaseous fission products near $\mathrm{UF}_{6}$ and the high reduction potential for fluorinating agents. This shows that a galvanic cell to separate $U_{6}$ is very feasible. The effect of activity coefficients for UNF components in the molten salt on electrochemical equilibrium potentials was determined to be negligible. 
The electrochemical potentials for all reactions for molten salt constituents and UNF constituents with low equilibrium potentials such as lathanides, alkali metals, and alkali earth metals were calculated and showed that a mixture of $\mathrm{LiF}-\mathrm{CaF}_{2}$ may be required to separate the lanthanides by electroplating. The $\mathrm{NaF}$ and KF components of UNF could be reduced before the lanthanides before the lanthides. The analysis of electrochemical potentials for the oxide reduction also showed that $\mathrm{LiF}_{-} \mathrm{CaF}_{2}$ would be an effective electrolyte allowing recovery of all UNF components.

A custom electrochemical reactor and electrodes have been fabricated for electrochemical fluorination (Figure 3). A system for reactor operation is nearing completion and will be used to prove the feasibility of the reactions in early FY13. The system is capable of operating the reactor with variable gas composition and pressure, while allowing recovery of the $\mathrm{UF}_{6}$

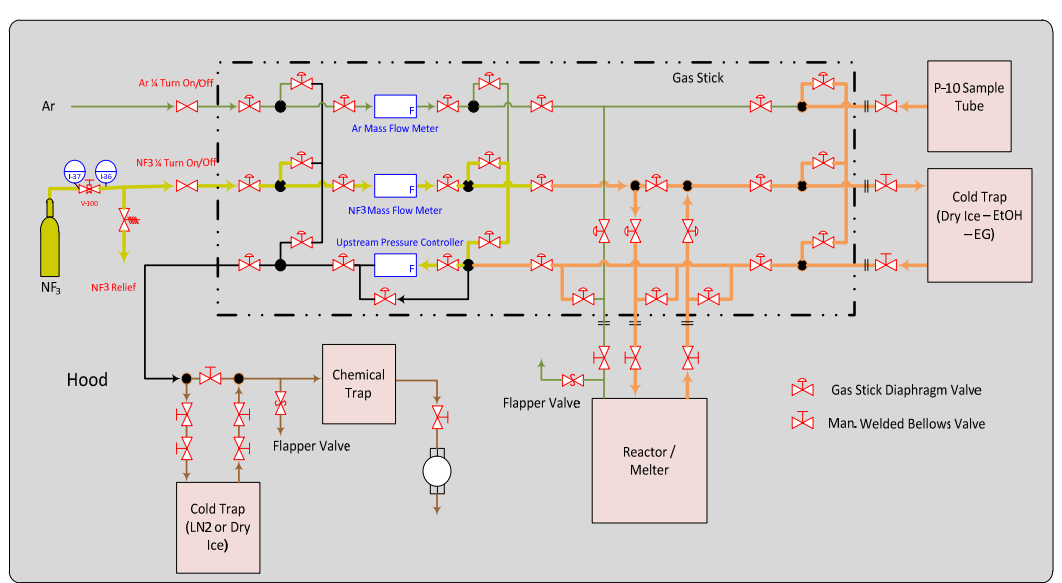

Figure 3. Reactor system for electrochemical fluorination. product.

The project has provided promising results for the feasibility of electrochemical fluorination and the development of a processing scheme that can start with oxide UNF. The project has also led to the submission of patent applications and full ARPA-E proposal invitations in addition to providing fundamental analysis of the process and constructing a system for proof of concept experiments. DOE and international groups working on pyroprocessing concepts have been engaged to include novel concepts from this project in roadmaps for UNF reprocessing. This included developing relationships with the University of Wisconsin and attending an international pyroprocessing conference at Argonne National Lab. The work on this project and equipment from the project provided a foundation for SRNL to get a $\$ 3.8$ million SunShot proposal on high temperature corrosion in molten salt heat transfer fluids. NEUP and FCRD proposals have also been developed relating to these concepts.

\section{Characterization of Chemical Changes Observed for Uranium and Plutonium Particulate Materials (N. J. Bridges, G. A. Fugate, M. R. Kriz, J. J. Pittman, M. J. Siegfried, M. S. Wellons)}

Abstract: Small uranium and plutonium particulates emanating from nuclear fuel cycle and weaponization processes can be collected at significant distances from their source. The chemical and physical characteristics of these particles depend on the production environment, while their chemical stability is determined by internal reactions with themselves, other environmental conditions and matrices upon which they settle. Therefore, a greater understanding of dispersed uranium and plutonium bearing particulate chemistry will result in more accurate fate and transport models, design of enhanced collection systems, and improved analytical characterization methodologies. Initial studies 
have focused on production of uranyl bearing particulates and the chemical changes that occur in the model system, uranyl hydroxide, when exposed to environmental stress.

The objective of this research is to gain a fundamental understanding of the species and phases formed when uranium- and plutonium-bearing particulates are exposed to environmental stresses. Knowledge of the fate and transport of uranium and plutonium particulate species is surprisingly limited considering the importance of this subject to the nuclear community. Health physics considerations related to lung deposition exclusively consider these compounds to be high fired oxides for lung deposition studies, while environmental or non-proliferation studies tend to study the materials radiometrically and/or destructively with no consideration of chemical speciation. This investigation has primarily addressed chemical characterization of these particulates, and will continue to concentrate on characterization of uranium and plutonium particles similar to those originating from nuclear fuel cycle and/or weaponization processes. The improved knowledge of chemical speciation can be used for Nuclear Non-Proliferation Treaty verification to design improved collection systems, development of enhanced dissolution and separation methodologies, and/or specific analytical measurements to allow more accurate determination of source term species and isotopic ratios.

During 2012 a sonic nebulization technique for producing uranium particulates from commercially available simple uranyl materials has been developed. Samples were collected passively on a variety of substrates and actively, when analytical techniques required production of a higher sample load. Simple substrates, such as silicon and gold coated silicon, were used to passively collect particles with minimal reactivity. After production, representative samples were stored under a variety of controlled temperature and relative humidities and analyzed periodically over the course of weeks to months. Other substrates, to be utilized in 2013, will consist of prevalent construction (e.g. steel, concrete) or industrial environment (e.g. iron oxides) related materials which represent a variety of surface reactivities and are probable sampling surfaces in the real world.

The chemical nature of the particulate material was analyzed using Raman spectroscopy to study the chemical species present in the sample. In particular, the $\mathrm{U}=\mathrm{O}$ bond is a useful probe for spectroscopic techniques, as it is sensitive to the coordination environment around the uranyl species. The particle morphologies for uranium particulates can be examined directly by scanning electron microscopy (SEM) and, as appropriate for smaller particles, by transmission electron microscopy (TEM). SEM images were collected using an instrument that combines the capability of doing high resolution SEM and elemental analysis using a large area energy dispersive X-ray spectroscopy, with the ability to do high resolution Raman spectroscopy simultaneously on the sample when desired. TEM grids were deployed as passive substrates for collection of particles and subsequently analyzed at Clemson University to detect low level uranium. In addition, a fraction of these samples are being analyzed using high resolution imaging and electron diffraction patterns of single particles after exposure to environmental stresses to detect phase changes. Macroscopic phase character of samples were determined using powder X-ray diffraction (XRD), with emphasis on attempting to detect potentially unique structures that may be formed based upon metastable structures formed during reactions of crystalline materials. 
Initial work during 2012 has shown that the interactions of the uranyl species with the salts studied to date appear to be weak and that formation of a hydroxide species generally occurs. The current particle generation equipment typically produces materials in the 5-10 micron range. Ongoing studies are being performed to focus on determining conditions necessary to produce target simple salts for followon work. Hydroxide particulates tend to be highly hydrated even under production conditions intended to produce very dry materials. The particle depicted in the SEM image within Figure 1 is a hydroxide particle that was passively collected as a wet particle, as evidenced by the faint ring around the now crystalline agglomerate. These "wet" particulates where produced under conditions that typically generate dry particles in other model systems. The uranyl hydroxide species formed in these processes tend to form macro to micro crystalline materials in a matter of days. Raman spectroscopy (Figure 1) suggests that the materials transition to another uranyl species as rapidly as 1 week (95\% relative humidity) while lower humidity studies where still reactive after several weeks. Although the Raman bands can only tentatively be defined as associated with uranyl hydroxide, powder XRD measurements suggest the initial material is consistent with metastudtite (a di-hydrate uranyl phase with the formula $\mathrm{UO}_{4} \cdot 2 \mathrm{H}_{2} \mathrm{O}$ ) whilst the most aged samples particles are consistent with studtite (a tetra-hydrate uranyl phase with the formula
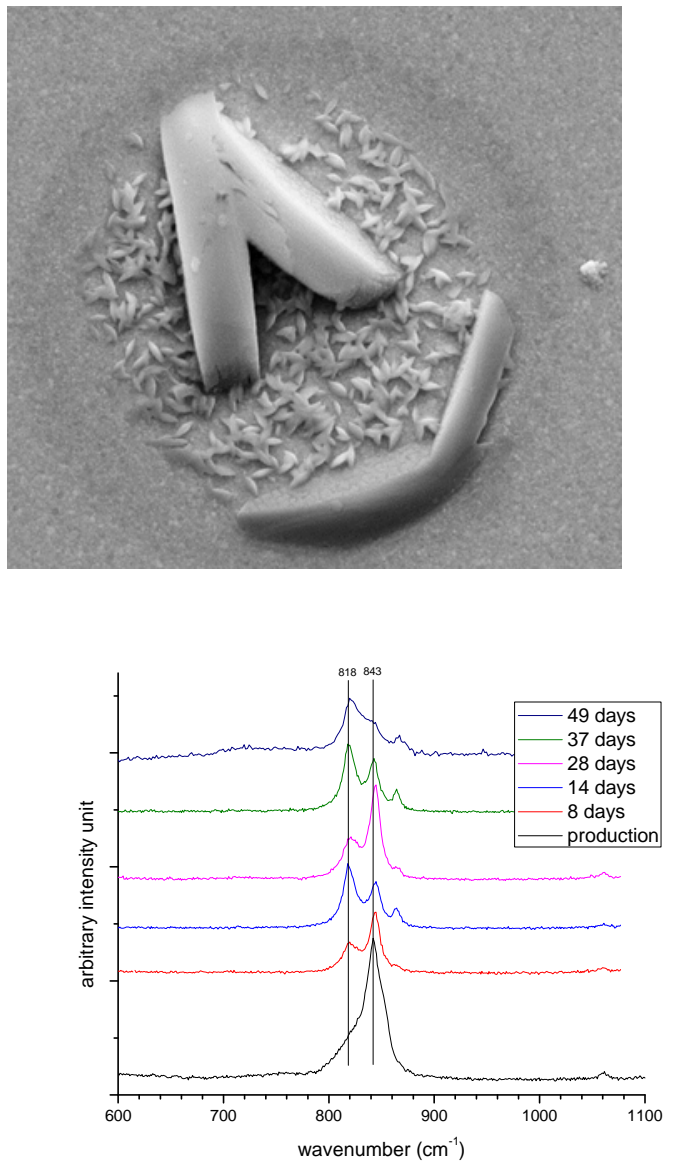

Figure 1. SEM image of uranyl hydroxide on gold stored at $\mathbf{7 5 \%}$ relative humidity for $\mathbf{2 8}$ days with associated Raman spectra showing transition between various hydration states. $\mathrm{UO}_{4} \cdot 4 \mathrm{H}_{2} \mathrm{O}$ ). Additional analyses (fluorescence, TEM, Auger electron, and others) are planned to confirm these results.

To date, the experimental results show changes in the chemical species that are consistent with expected results for the imposed environmental insult. Follow-on work during 2013 will focus on additional uranyl salts and a single plutonium salt system(s), as well as additional particulate production equipment able to produce desired materials in the 1-5 micron range. 
Unveiling micro-to-nano fluidics physico-chemical phenomena for advance separation and ultra-sensitive detection of nuclear species (P-S. Lam, M. G. Bronikowski, A. E. Méndez-Torres)

Abstract: The state-of-the-art technologies based on fluidics with the characteristics of electrokinetics and electrophoresis are used to develop advanced separation processes for highly radioactive actinides and isotopes, ultra-sensitive sensors capable of detecting cementitious barrier materials degradation ion species and radionuclides migration in groundwater, as well as chemical and biological warfare agents. This work will provide cross-cutting solutions in multiple thrust areas such as fate and transport of radionuclides and chemical processing. There are two main research areas: 1) advance separation and speciation of special nuclear materials with microfluidics; and 2) nanofluidics physics and chemistry for highly selective sensing and speciation of radionuclides. Ultimately, micro/nanofluidics will be integrated in a Lab-on-a-Chip (LOC) that allows the capabilities for separation and detection up to million-folds. This project integrates SRNL expertise on fluidics, nuclear-physics, separation, concrete, modeling, and nanofabrication.

The technical objective of this proposal is to investigate and explore the underlying science and physics of microfluidics with the aim to establish SRNL in-house fluidics capabilities by adopting a "lab on a chip" (LOC) approach with multifunctional and crosscutting applications in nuclear material separation technologies and in developing trace detection sensor systems for toxic chemicals and radionuclides. For highly radioactive separations, the small size decreases ALARA concerns and permits much smaller footprints. The laminar flow in the microchannels of these chips maximizes the efficiency in molecular diffusion in an aqueous-organic liquid extraction and separation. The small sample size in microfluidics also results in fast mixing and equilibration, reduced reagent consumption, and better temperature control, facilitate the use of less stable solvents, reductants, and complexants. In sensor development with micro- and Nano fluidics, the electric double layer effects enable higher ion preconcentration which is essential for ultra-sensitive devices. This type of sensors has a potential for multiplex assay and detection for multiple analytes in parallel operation, and can be customized for measuring many targeted analytes, such as toxic chemicals and radionuclides (e.g., chloride, sulfate, Magnesium ions, nitrate, tritium, $\mathrm{C}-14, \mathrm{Ni}-59$, and $\mathrm{Tc}-99, \mathrm{Pu}, \mathrm{U})$, proteins and DNA at various sampling station.

Two Lab-in-a-Chip Microstations are used for this project. One Microstation will eventually be deployed to the SRNL limited area for nuclear material separation. These two Microstations allow simultaneously work on separation science and on sensor development with different chips tailored for each application. A numerical modeling capability is established for benchmarking experimental results, upon which the numerical experimentation can be performed for down-selecting chip specifications or for micro/nano-channel design/fabrication. In addition, the work fully utilize the SRNL existing facilities, such as Nuclear Magnetic Resonance (NMR), Dual Laser 3-D Micro Raman, 2-D IR, and Micro IR with environmental cell, SEM with Energy Dispersive Spectroscopy, XRD, and Mass Spectrometer.. The project team will 1) identify equipment and computation/modeling needs to establish the SRNL Fluidics Laboratory; 2) procure and install the equipment in the laboratory; 3 ) conduct functional testing of equipment on cold surrogates; 4) Conduct theoretical modeling for the design of micro/nano-channels to achieve high pre-concentration for ultra-sensitive trace detection and monitoring, and for improving the LOC performance in nuclear material separation, 5) explore micro/nano-fabrication technologies for 
manufacturing customized $L O C, 6)$ initiate $\mathrm{Pu} / \mathrm{U}$ analogous studies using surrogates, 7) initiate the development of small scale prototype for a deployable system; and 8) seek external funding for on-going development. The work will lead to the roadmap for operation with radioactive (actinide) solutions and hazardous or biological species, and position SRNL for seeking external funding for further development in fluidics applications.

A Dolomite workstation has been installed in SRNL to conduct task on nuclear material separation. The preliminary system testing is in progress. The system has shown the capability of separating two materials with two distinct densities in the form of bubbles. Figure 1 shows a sequence of air droplets being made in a 190 micron Dolomite T-chip during initial testing of the equipment after a flow resister was placed in the air line.
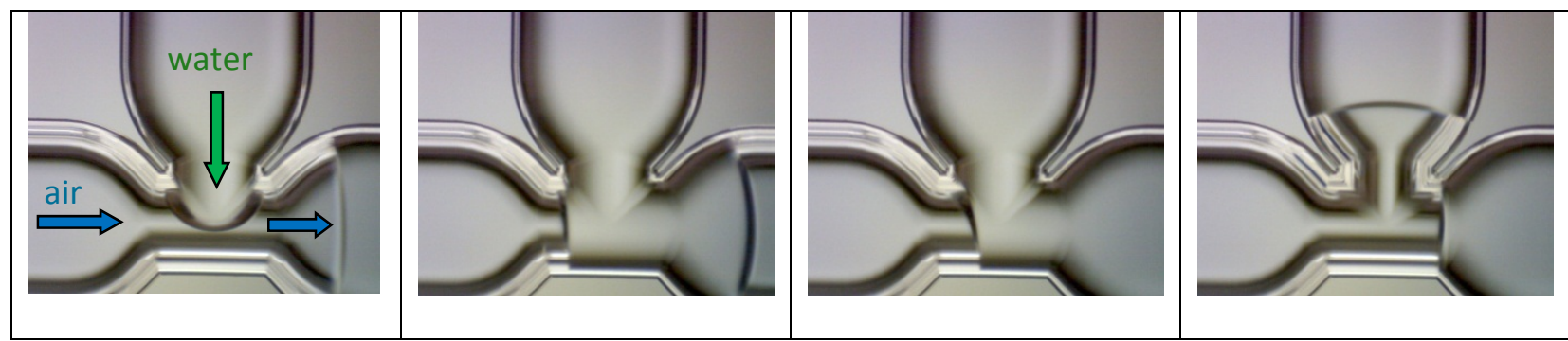

Figure 1 Sequence of air bubbles being formed in microfluidics channel of Dolomite System.

The LabSmith workstation was set up in Hydrogen Technology Research Laboratory (HTRL) for sensor developing. Current effort is focused on visualization and quantification of ion concentration in fluid using dye as a surrogate. The system can be seen in Figure 2 with fluorescent image of a microchannel. The microchannel can be used with SRNL/MS\&T equipment, 3D Confocal Micro Raman Spectroscope to reveal detailed concentration distribution of a chemical species in a subsurface microchannel.

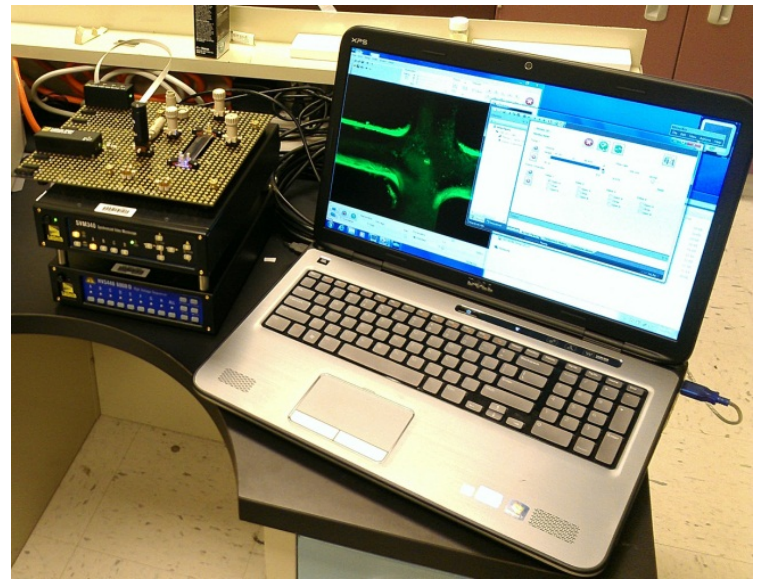

Figure 2. LabSmith System and fluorescence microscope showing dye passing through a microchannel.

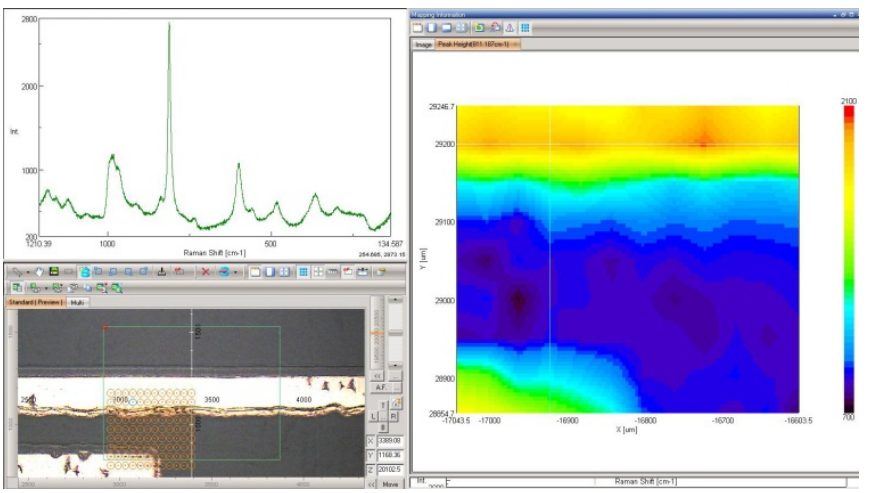

Figure 3. LabSmith chip with 3D Confocal Micro Raman spectroscopy showing concentration intensity of the materials. 
Preliminary results show that organic-aqueous separation is achievable with the Dolomite System, and ion concentration can be detected with the LabSmith workstation. The Raman-fluidics result indicates the potential for developing high fidelity optofluidics devices for the identification of radioactive species in aqueous media. Utilizing spectroscopy techniques also allows the development of nanofluidics scintillation systems for the detection of tritium and other radioactive species.

\section{LDRD-2012-30 Hardware in the Loop Electrical Grid Simulator (J. V. Cordaro, G. H. Fisher Jr., J. R. Coleman)}

Abstract: While there are numerous locations that can model and perform grid simulation, the combination of actual electrical hardware and grid simulation with Hardware-in-the-Loop (HIL) capability is rare. By having HIL capability, high voltage and high current switchgear can be tested and models can be generated to duplicate actual performance. With the modernization of the US electrical grid, high accuracy current measurements are essential at key power distribution points. The Savannah River National Laboratory (SRNL) has the nation's only High Current Calibration Laboratory (HCCL). With high current standards originally from NIST, the HCCL is a national asset. Models are needed of the precision current sensors used in the HCCL and how their performance compares to commercial current sensors used on the grid.

The objective of this research is to build and validate high frequency models of the NIST fabricated Rogowski coils located in the HCCL and compare performance to commercial Current Transformers (CT). Currents from hundreds of amps to 100,000 Amps will be applied to the sensors in series with high speed data capture. Frequencies ranging from $\mathrm{DC}$ to $2 \mathrm{KHz}$ will be generated. The effects of adding multiple conductors through a CT to simulate high currents will be evaluated and compared to single conduction measurements. Based on actual data, models will be validated using a Real Time Digital Simulator (RTDS). This project supports SRNL's involvement in the 15 Mega Watt Electrical Grid Simulation Laboratory being built at the Clemson University Restoration Institute located in Charleston SC. This lab will be the highest power grid simulation laboratory in the world.

During 2012, a state-of-the-art RTDS was selected, specified and purchased. The RTDS was installed in the SRNL HCCL. Acceptance and startup testing of the RTDS was completed. SRNL researchers completed training building custom models of electrical components using the RTDS software. Testing was completed using single analog output/inputs and capture using on the RTDS. SRNL researches completed literature searches and selected the highest accuracy CT's for evaluation. Three 10,000 Amp CT were purchased from Zimmer in Germany and received. Due to the potentially high magnetic fields that can be generated in the HCCL, a detailed hazardous analysis was completed and approved clearing the way for research to begin. Additional power amplifiers were installed in the HCCL to reach 1000 Amps continuous output.

Figure 1 shows the connection of the two NIST Rogowski coils installed in a custom Faraday Cage being fed current from a pulse 100,000 Amp transformer. The analog outputs from the Rogowski coils are connected to integration and a high speed data acquisition system. This setup allows simultaneous high speed capture of the two NIST coils allowing inter-comparison data. 
Figure 2 shows the connection of the Rogowski coils being fed from a series of high current amplifiers that can provide continuous 1000 Amps from DC up to a frequency of $2 \mathrm{KHz}$.

Figure 3 shows how using the RTDS, time synchronized pulses can be generated driving the high current transformer feeding current through the Rogowski coils with the output of the coils connected back into the RTDS.

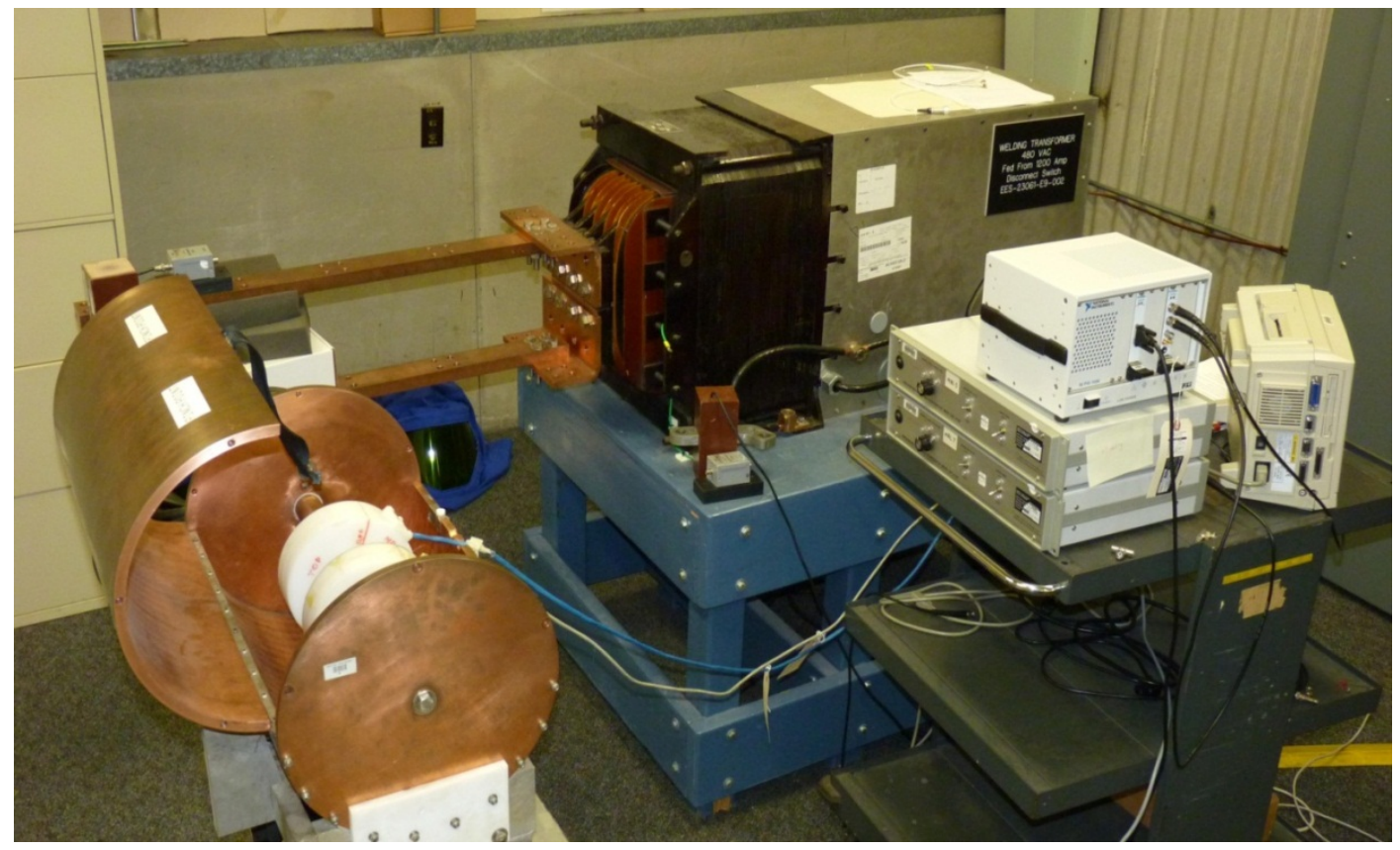

Figure 1: High Current 100,000 Amp transformer with Faraday Cage and Rogowski Coils

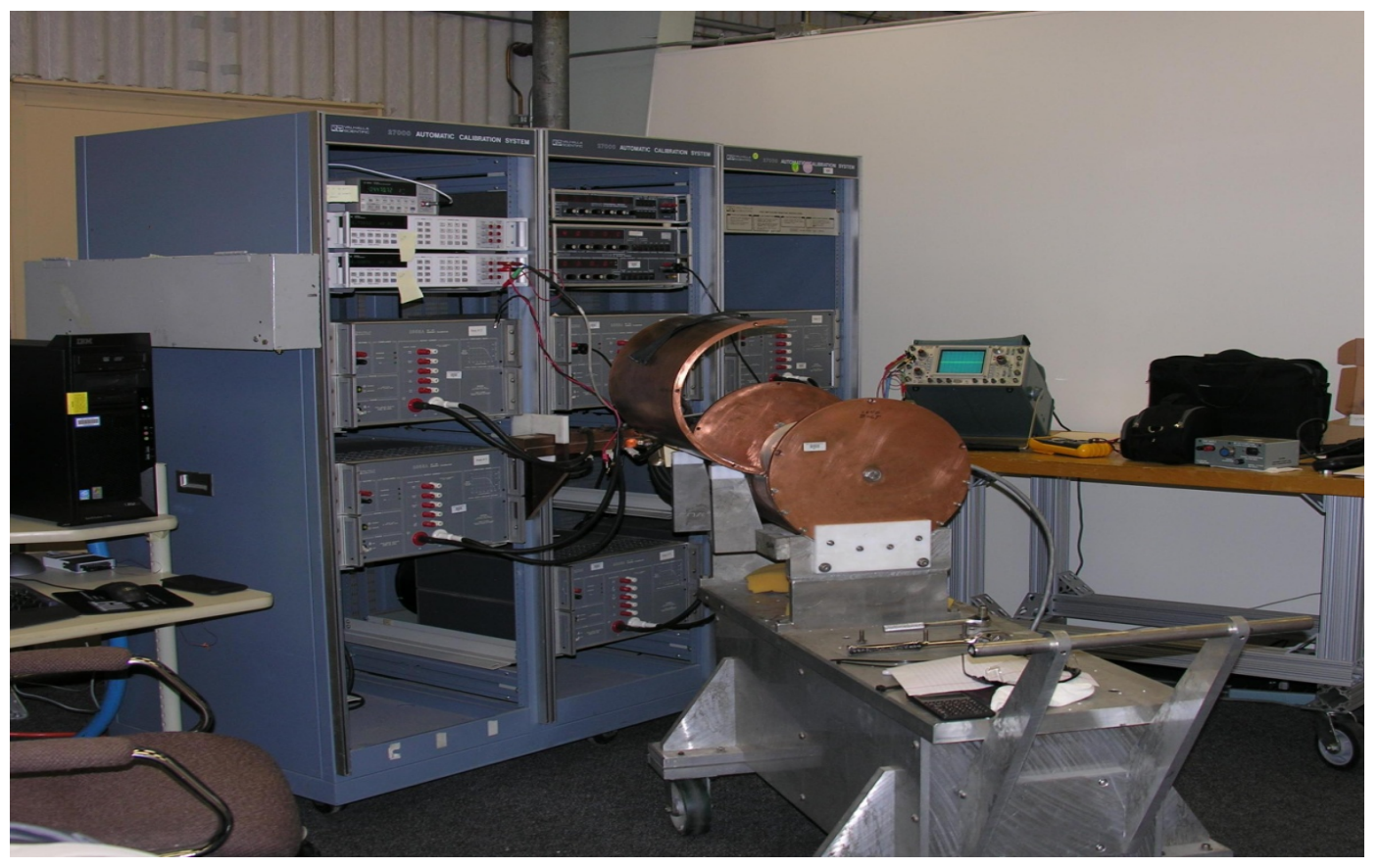

Figure 2. 1000 Amp Continuous Power Amplifiers with DC to $2 \mathrm{KHz}$ Response 


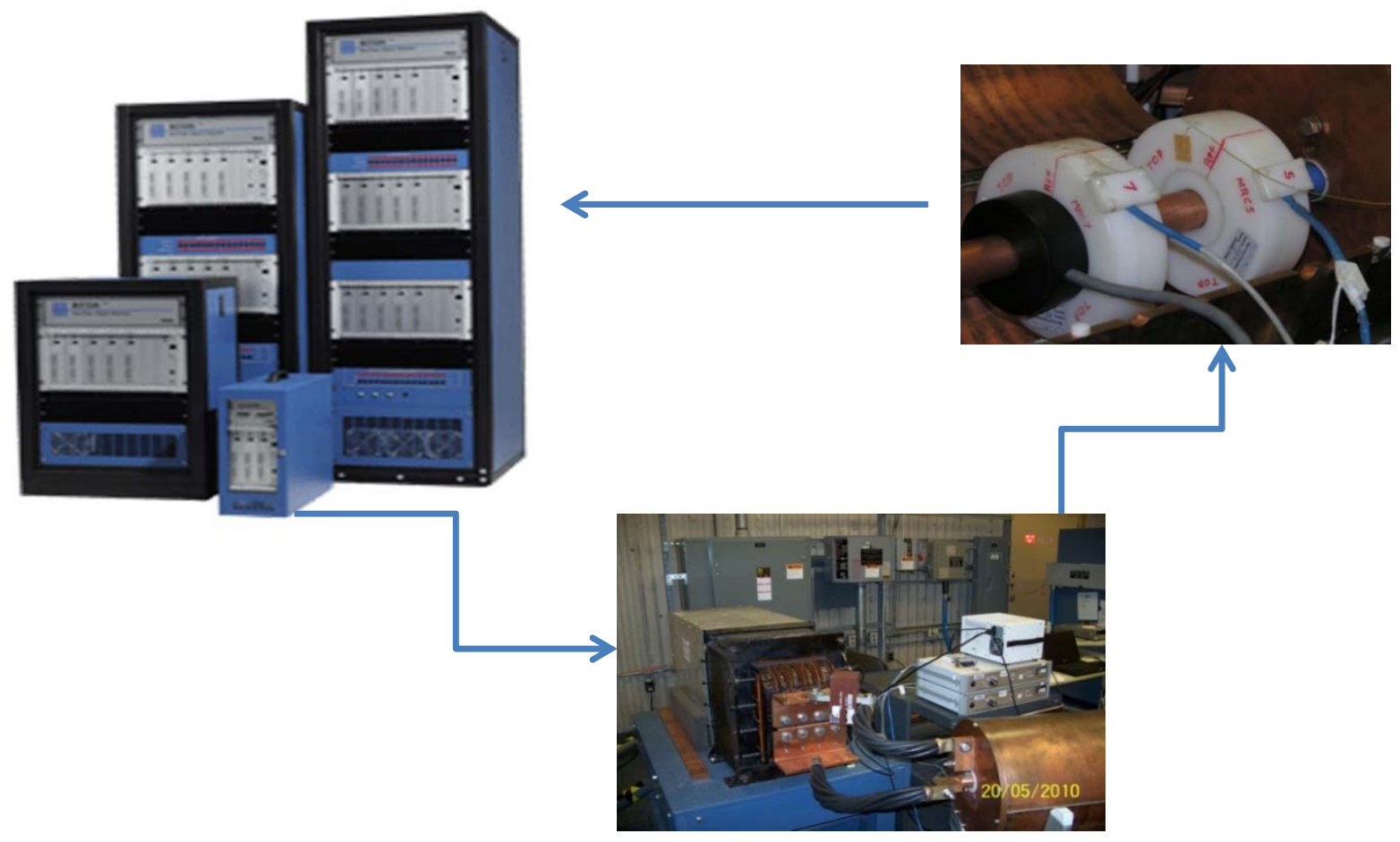

Figure 3. Hardware-In-the-Loop Connection with a RTDS

Beginning in FY2013, models will be created using the RTDS software. An example is shown in Figure 4. One the equivalent circuit of the high current device is known; the modeling software will perform the integration and convert to a format used for real time simulation. By running current through the high current sensors and simultaneously running a virtual simulation of the model, the output data can be compared and the model parameters adjusted accordingly to fit actual performance. A paper will issued documenting the results including the design of the models of the CT's and Rogowski coils from DC to 2 $\mathrm{KHz}$.

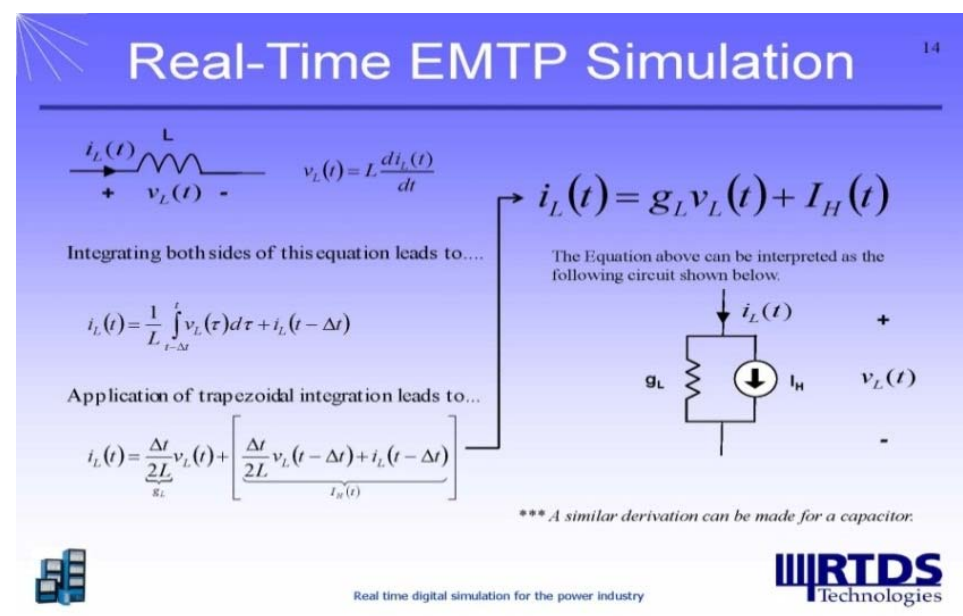

Figure 4. RTDS Modeling Software 
The RTDS software includes models for 1000's of devices that allow the design of complex grids. Using the models SRNL developed for the commercial high current CT's, equipment manufacturers and power companies can utilize the data and models created to better understand the performance of the CT's under actual condition on the nations electrical grid.

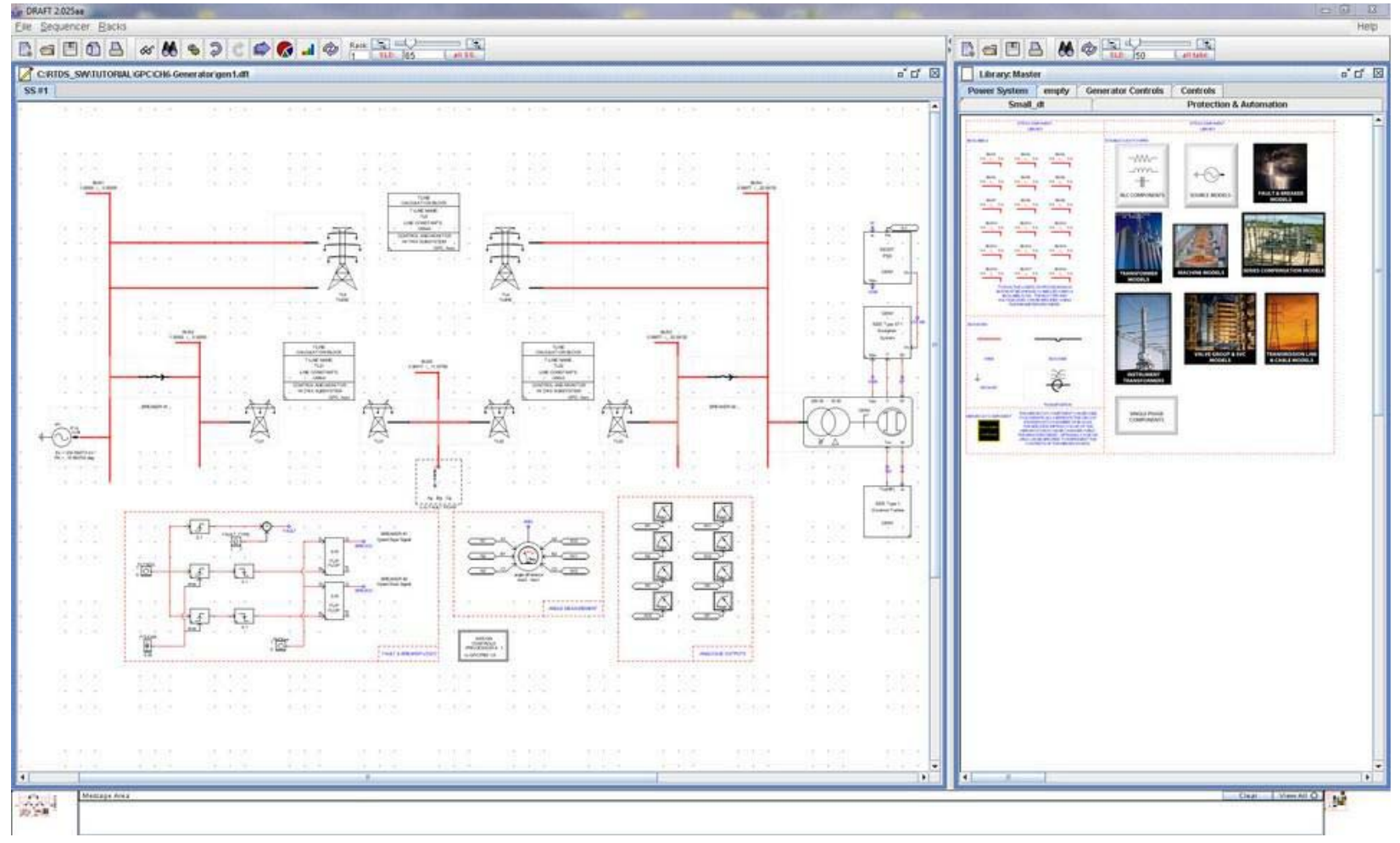

Figure 5. Virtual Electrical Grid

\section{FY 2012 Standard Project Summaries}

\section{Tunable Hydrophobic/philic Reaction Sites on Multifunctional Catalysts for the Redox of Lithium for Improved Energy Storage (E. B. Fox (PI), R. E. Fuentes, S. Kendrick, H. R. Colón-Mercado)}

Abstract: Li/air batteries are of considerable interest for energy storage due to the high charge transfer that occurs during the oxidation-reduction reactions of lithium. By increasing the kinetic rates of reaction, it is possible to reach power densities closer to the theoretical value of $13 \mathrm{Wh} / \mathrm{g}$ in a Li/air system. Since only the active anode material is needed in the construction, lighter weight construction and smaller cell size are possible, benefiting portable applications. The forward reaction is referred to as the Oxygen Reduction Reaction (ORR) and the reverse reaction is called the Oxygen Evolution Reaction (OER). The reversibility of the redox process is hindered by the formation of insoluble lithium oxide species (Eq 1 and 2) in a non-aqueous environment, which in turn hinders the dissociation of evolved oxygen species. To overcome these challenges, the oxidation-reduction of $\mathrm{Li}_{2} \mathrm{O}$ and $\mathrm{Li}_{2} \mathrm{O}_{2}$ will be catalyzed at room temperature by the use of novel, dual-functional catalysts with targeted reaction sites. 
Lithium/air batteries are considered the ultimate power source among electrochemical energy conversion and storage cells derived from lithium chemistry ${ }^{1}$. With a theoretical open circuit voltage close to $3 \mathrm{~V}$ and a theoretical specific energy of $13 \mathrm{kWh} / \mathrm{kg}, \mathrm{Li} /$ air batteries have recently gained considerable interest for energy storage due to the high charge transfer that occurs during the oxidation-reduction reactions of lithium. These batteries are unique in that the cathode active material (oxygen) is not stored in the battery. Depending on the discharge currents and electrolyte composition for the non-aqueous system, the discharge of the battery will result in the redox reaction of Li to lithium peroxide (Eq. 1) followed by the further reduction to lithium oxide (Eq. 2). The overall reaction voltage is given by (Eq. 3) ${ }^{2}$.

$$
\begin{array}{lll}
2 \mathrm{Li}+\mathrm{O}_{2} \leftrightarrow \mathrm{Li}_{2} \mathrm{O}_{2} & \mathrm{~V}=3.10 \mathrm{~V} \\
2 \mathrm{Li}+\mathrm{Li}_{2} \mathrm{O}_{2} \leftrightarrow 2 \mathrm{Li}_{2} \mathrm{O} & \mathrm{V}=2.72 \mathrm{~V} \\
4 \mathrm{Li}+\mathrm{O}_{2} \leftrightarrow \mathrm{Li}_{2} \mathrm{O} & \mathrm{V}=2.91 \mathrm{~V}
\end{array}
$$

The discharge (forward) reaction is referred to as the Oxygen Reduction Reaction (ORR) and the charge (reverse) reaction is called the Oxygen Evolution Reaction (OER). In a typical system the cathode consists of metal catalyzed, carbon-based, porous gas diffusion media (GDM) that enhances the oxygen diffusion into the system. The metal catalyst is needed in order to enhance the formation of lithium oxide from the lithium peroxide and to carry out the OER in order to recharge the battery.

Currently, the reversibility of the redox process is hindered by the low solubility and precipitation of the lithium discharge products (Eq 1 and 2). These insoluble oxides passivate the catalyst surface, eventually hindering the ORR ${ }^{3}$. In addition, precipitates block gas channels leading to oxygen mass transport resistance which limits the cells to low operating current densities ${ }^{4}$. The introduction of atmospheric air adds parasitic reaction species, such as humidity, which can

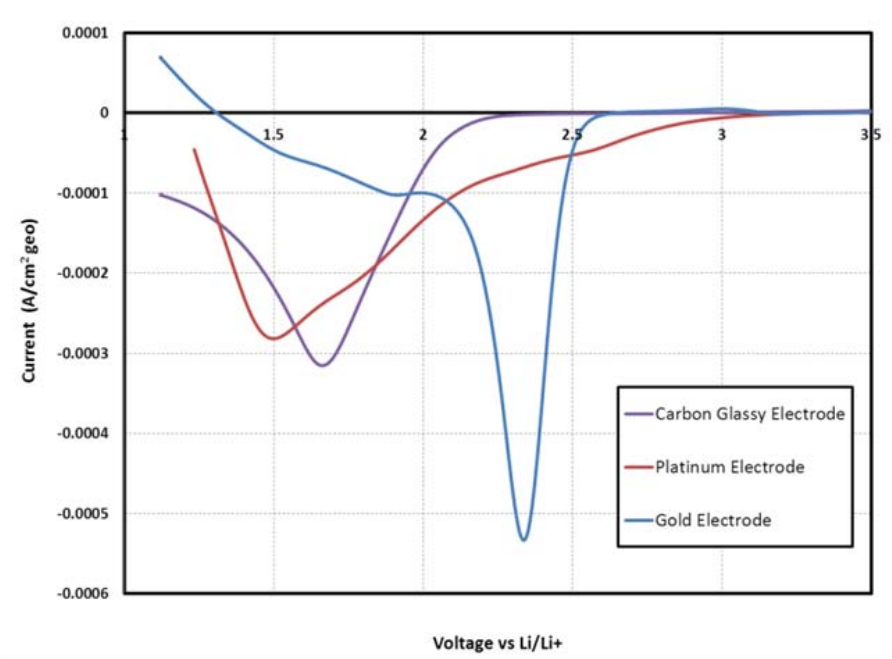

Figure 2. Voltammogram of different electrodes. Current is normalized by the surface area of electrode.

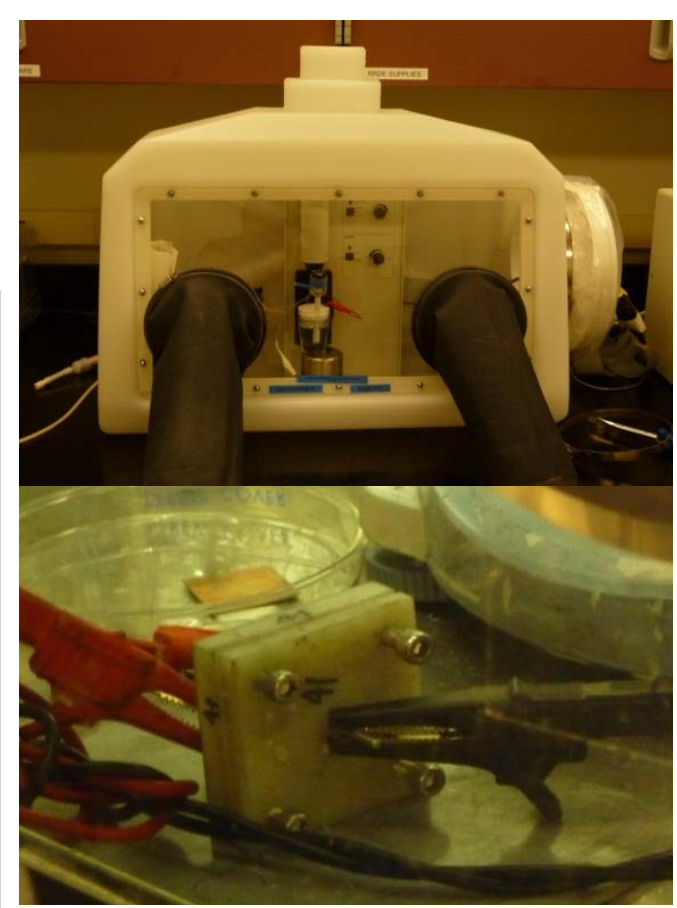

Figure 1. a) RDE set-up in a dry, portable glovebox unit b) checking the open circuit voltage on an assembled battery. 
diffuse and oxidize the lithium anode. Carbon dioxide can react with the lithium oxide discharge product and form lithium carbonate rendering the battery irreversibly damaged. Because of the lack of fundamental understanding of the reaction mechanisms within the cell system, theoretical limits have not been reached in either the ORR or OER.

Performance for the oxygen reduction reaction (ORR) was obtained using electrochemical techniques in a rotating disk electrode system. The setup consisted of a BASi RDE-2 rotator system which has the traditional three electrode configuration. To prevent moisture, the rotator system was enclosed inside a plastic glovebox with a continuous flow of dry argon. The rotator system was connected to a BASi potentiostat which was connected to a computer. The software Epsilon from BASi was used as the interface to obtain electrochemical data. The electrodes used consisted of a platinum coil as the counter electrode, a carbon glassy electrode or platinum electrode as the working electrode with $3 \mathrm{~mm}$ diameter and an E-DAQ hydrogen electrode as the reference electrode. The E-DAQ hydrogen electrode was used instead of Li foil because of instability during long term operation and safety concerns. The reference potential was 2.72 vs. $\mathrm{Li} / \mathrm{Li}+$ obtained in a solution of $1 \mathrm{M}$ Lithium bis(trifluoromethylsulfonyl)imide (LiTFSI) in tetraethylgylcol dimethylether (TEGDME). This electrolyte was prepared inside a glovebox and molecular sieve was placed 24 hours prior to use to reduce moisture content. The LiTFSI and TEGDME were purchased from $3 \mathrm{M}$ and from Sigma-Aldrich, respectively. Cyclic voltammetry experiments ran between 1.15 to $3.90 \mathrm{vs}$. Li/Li for 10 cycles first in a $\mathrm{N}_{2}$ bubbled solution and then in an $\mathrm{O}_{2}$ bubbled solution at a scan rate of $100 \mathrm{mV} / \mathrm{s}$. The data from the $\mathrm{N}_{2}$ bubbled solution was used to correct for the background current for the performance of ORR in the $\mathrm{O}_{2}$ bubbled solution. Experiments were performed using the working electrodes without any coating and with a layer of catalyst. To coat the electrode, carbon inks were prepared with $8 \mathrm{mg}$ of the carbon or carbon with catalyst dispersed in $4 \mathrm{~mL}$ of deionized (DI) water and $4 \mathrm{~mL}$ of ethanol. A volume of $3.5 \mu \mathrm{L}$ was placed on top of the electrode corresponding to one drop. After it dries $2 \mu \mathrm{L}$ of a solution of $0.2 \%$ $\mathrm{w} / \mathrm{w}$ of Nafion in Ethanol was added. All of the experiments ran at room temperature.

An example of the RDE produced throughout this project is presented left in Figure 2. The figure compares the performance for ORR of three different planar electrodes: carbon glassy, platinum and gold. As seen in the voltammogram the platinum electrode exhibits activity at a lower potential than the other two electrodes. However, when it reaches peak current density it is similar to the glassy carbon electrode at a similar potential. It is also observed that the highest peak current corresponds to gold electrode. A stateof-the-art catalyst that can be highly

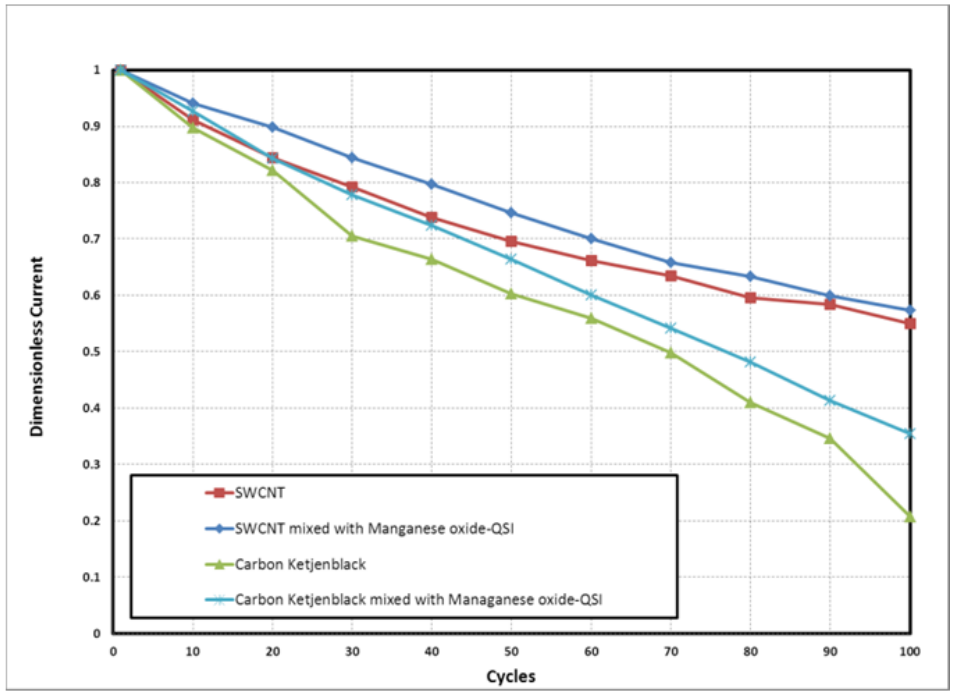

Figure 3. ORR peak current during cycling for single wall carbon nanotubes and carbon Ketjenblack pure and mixed with $\mathrm{MnO}_{2}-\mathrm{QSI}$. The current was normalized by the amount of carbon and divided by the current at the $1^{\text {st }}$ cycle. 
active can combine Pt-Au as previously reported ${ }^{5}$. The problem is that using expensive precious metals can add costs into the process of Li-Air batteries making them unfeasible to produce commercially.

Electrochemical activity can also be provided with different types of carbon with high surface area. In Figure 3, it is presented the cyclability of two types of carbon materials: Single Wall Carbon Nanotubes (SWCNT) and Carbon Ketjenblack. The current obtained was normalized by the mass of carbon added and divided by the current at the first cycle to provide peak current incremently every 10 cycles for 100 cycles. The two carbon materials were also mixed with Manganese Oxide from Quantum Sphere Inc. (QSI) with corresponding $50 \mathrm{wt} \%$ as a catalyst for ORR. Ketjenblack has highest losses between cycles even though it has a higher surface area than SWCNT. Comparing the carbon materials mixed with $\mathrm{MnO}_{2}$, there is a slightly increase of peak current activity. In terms of cyclability, it does not seem there was an improvement. Since carbon provides high activity for the ORR, the challenge remains in a catalyst that can increase considerably the peak current density and cyclability for the Li-Air cell. This highlights the complexity in designing electrodes and electrolyte selection for Li/air applications.

1. Kumar, B.; Kumar, J.; Leese, R.; Fellner, J. P.; Rodrigues, S. J.; Abraham, K. M., A Solid-State, Rechargeable, Long Cycle Life Lithium-Air Battery. J. Electrochem. Soc. 157 (1), A50-A54.

2. Zhang, S., Discharge characterisitics of a non-aqueous electrolyte $\mathrm{Li} / \mathrm{O}_{2}$ battery. J. Power Sources 2101, 195, 1235-1240.

3. Lu, Y.-C.; Gasteiger, H. A.; Crumlin, E.; Jr., R. M.; Shao-Horn, Y., Electrocatalytic activity studies of select metal surfaces and implications in Li-Air batteries. J. Electrochem. Soc. 2010, 157 (9), A1016A1025.

4. (a) Beattie, S. D.; Manolescu, D. M.; Blair, S. L., High-Capacity Lithium--Air Cathodes. J. Electrochem. Soc. 2009, 156 (1), A44-A47; (b) Laoire, C. O.; Mukerjee, S.; K.M., A.; Plichta, E. J.; Hendrickson, M. A., Elucidating the mechanism of oxygen reduction for lithium-air battery applications. J. Phys. Chem. C 2009, 113, 20127-20134.

5. Lu, Y.-C.; Xu, Z.; Gasteiger, H. A.; Chen, S.; Hamad-Schifferli, K.; Shao-Horn, Y., Platinum-Gold Nanoparticles: A Highly Active Bifunctional Electrocatalyst for Rechargeable Lithium-Air Batteries. Journal of the American Chemical Society 2010, 132 (35), 12170-12171.

\section{Redox Catalyst Development for Improving Electrolytic Cells (R. E. Fuentes,} H. R. Colón-Mercado, M. J. Martínez-Rodríguez)

Abstract: Unitized regenerative fuel cells (URFCs) are electrochemical cells working both as fuel cells (FC) and water electrolyzers (WE). In WE mode, the water is separated to hydrogen and oxygen which are stored to later be recombined to produce electricity in the subsequent FC mode. The key technology in the development of URFCs is the fabrication of a bifunctional electrode with an active electrocatalyst for both the oxygen reduction reaction (ORR) and the oxygen evolution reaction (OER) at the oxygen electrode. Because unsupported Pt group metals (PGM) are used as the bifunctional electrode, cost is a major impediment towards this technology. The team led by the Savannah River National Laboratory (SRNL) developed nanostructured supported PGM catalysts. The prepared catalysts consist of high 
surface area Pt-Ir-Co on conductive titanium carbide (TiC) support capable of increasing the round trip efficiency from $55 \%$ to $62 \%$.

Unlike batteries, URFC have advantages such as being free of self-discharge and theoretically high energy densities. This makes URFCs a promising energy storage system for specialty applications, however because of the use of high loadings of platinum group metals (PGM) such applications are limited to space applications. Improvements in the area of bifunctional catalysts for URFC are a natural fit with the hydrogen storage program already in place at SRNL. In addition, this project has increased the interaction with international groups such as the National Institute of Advance Industrial Science and Technology (AIST) of Japan. In the present work, we prepared and demonstrated bifunctional electrocatalysts able to increase the round trip efficiency as compared to state of the art electrocatalysts.

Pt as an electrocatalyst is known to be active towards ORR, however, at high potentials it forms a nonconductive oxide. ${ }^{1}$ This makes it impractical to use as an electrocatalyst for OER by itself. Mixing Pt and Ir can be used as an oxygen electrode for URFC since Ir and its oxides are an active catalyst for $O E R^{2}$ and can provide a bifunctional mechanism. To synthesize the Pt-Ir electrocatalysts an ethylene-glycol colloidal procedure similar to the procedure published by Fuentes et al. was used. ${ }^{3}$ In brief, a solution of 0.2 $\mathrm{M} \mathrm{NaOH}$ in ethylene glycol was added

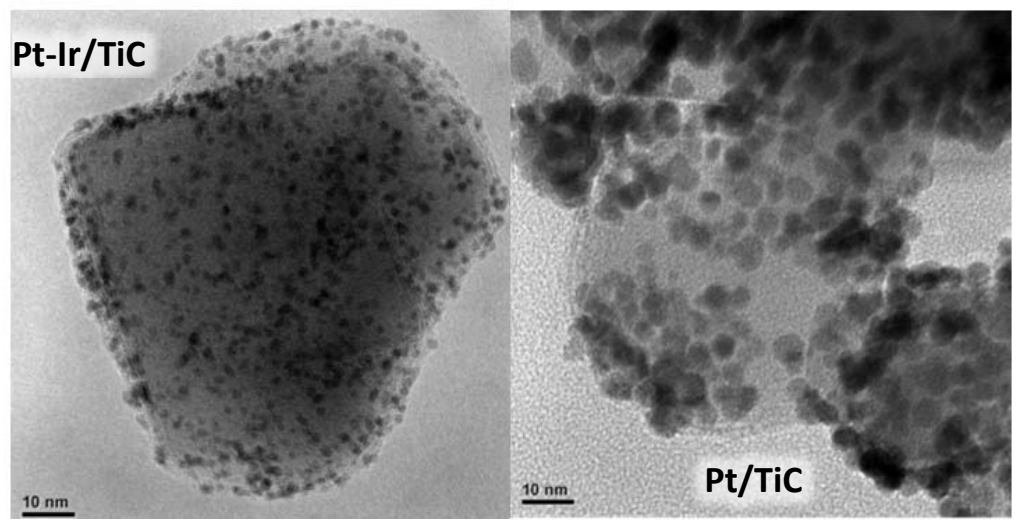
to a round bottom flask. The TiC support and the metal chloride salts $\left(\mathrm{H}_{2} \mathrm{PtCl}_{6}, \mathrm{H}_{2} \mathrm{rCl}_{6},\right)$ were added and mixed for approximately $2 \mathrm{~h}$. The homogeneous mixture was subsequently heated to $180^{\circ} \mathrm{C}$ for $3 \mathrm{~h}$. The sample was mixed with approximately $300 \mathrm{~mL}$ of deionized (DI) water, and hydrochloric acid was added until a $\mathrm{pH}$ of 1 . The sample was filtered, washed and dried at $80^{\circ} \mathrm{C}$ under vacuum conditions overnight. Electrochemical measurements were conducted in a standard three electrode electrochemical cell at room temperature using a rotating disk electrode (RDE) setup. A high resolution transmission electron microscopy (HRTEM) was used to image the lattice of the nanostructures and measure the particle diameter. X-ray powder diffraction was performed using a PANalytical X'pert Pro to characterize the products, using $\mathrm{Cu}-\mathrm{K}$ radiation. Compositional analyses of the catalysts were performed using inductive coupled plasma-emission spectroscopy.

The figure on the right shows the typical HRTEM image for Pt and Pt-Ir supported in TiC. The prepared catalysts show a particle size of 1-2 $\mathrm{nm}$ and 5-8 nm for Pt-Ir/TiC and Pt/TiC, respectively. The TiC support varies in dimensions between $20-50 \mathrm{~nm}$. The difference in particle size arises from modifications to the original recipe in order to adsorb the Pt nanoparticle on to the support. 
The ORR and OER activity of the electrocatalysts was measured electrochemically by obtaining linear sweep voltamograms in oxygen (ORR test) and nitrogen (OER test) saturated $0.1 \mathrm{M} \mathrm{HClO}_{4}$ solution. The figure on the left shows the specific activities of the different electrocatalysts through the kinetically limited section of the polarization curve. The curves below $1.2 \mathrm{~V}$ correspond to the performance for ORR and the curves above correspond to the performance for OER. The space between the curves corresponds to the kinetic inefficiencies of the bifunctional electrocatalysts. Therefore, reducing the gap indicates an improvement in the round trip efficiency. As observed, when using pure metal components such as in the case for Pt black (red solid line), the efficiency varies from $54 \%$ to $43 \%$ depending on the current density. However, when the catalyst is composed of bimetallic or tri-metallic as in the case of Pt-Ir-Co/TiC (dashed blue line), the efficiency increases to $62 \%$ and $58 \%$. In addition it is important to

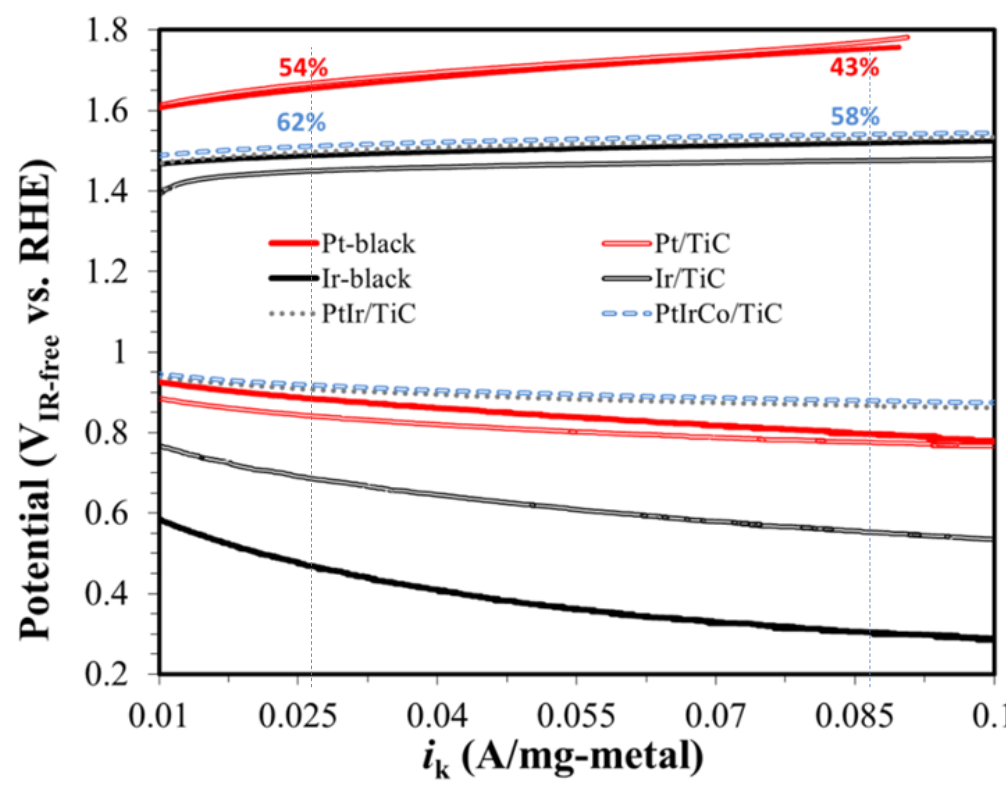
mention that the stability of the prepared electrocatalysts is comparable to that of commercial metal blacks.

In conclusion, stable and highly active Pt base electrocatalysts supported in a conductive support were synthesized and tested. Results showed higher efficiency due to their high specific activities. Future studies consist of testing the catalysts in a URFC system by our AIST partners.

1. Andolfatto, F., Solid Polymer Electrolyte Water Electrolysis: Electrocatalysis and Long-Term Stability. International Journal of Hydrogen Energy, 1994, 19 (5), 421-427.

2. Song, S., Electrochemical Investigation of Electrocatalysts for the Oxygen Evolution Reaction in PEM Water Electrolyzers. International Journal of Hydrogen Energy, 2008, 33, 4955-4961.

3. Fuentes, R. E.; Farell, J.; Weidner, J. W., Multimetallic Electrocatalysts of Pt, Ru, and Ir Supported on Anatase and Rutile $\mathrm{TiO}_{2}$ for Oxygen Evolution in an Acid Environment. Electrochemical and SolidState Letters, 2011, 14 (3), E5-E7.

\section{Implementation and Testing Organic Thin Film Systems as Beta Radiation Sensors} (A. L. Washington II, L. C. Teague, J. R. Coleman, R. J. Lascola (SRNL); J. Anthony (University of Kentucky))

Abstract: The objective of this project was to develop and test the charge transport phenomena in organic semiconductor materials fabricated in organic-based technologies such as organic-based field effect transistors (OTFT), organic based memory devices, energy storage materials, organic lightemitting diodes (OLEDs), organic-based photovoltaics (OPVs) and organic-based radiation detectors. 
This work specifically addresses how beta irradiation on these organic-based devices affects the charge transport and conductivity from the absorption of the molecules in the organic layer. Organic-based materials are becoming increasingly popular in the semiconductor field due to their potential for highperformance, low-power, low-cost alternatives to existing technologies, however, the ability to interact with ionizing radiation in the application of remote sensing is a multifaceted challenge that is new area of development that would contribute to the sustainability of organic-based technologies.

The overall objective of these studies was to investigate how beta irradiation affects the charge transport and charge trapping of organic thin film transistors (OTFTs) as well as if these materials would provide an electrical response in the presence of beta radiation. ${ }^{1}$ A change in the overall charge transport of the OTFTs is observed upon exposure to beta radiation which is similar to what is seen in the photon interaction of gamma irradiation as shown previously by the research community. ${ }^{2}$ Through MCNP modeling and experimental exposures, the beta interaction has been investigated on OTFTs of various atomic components to ascertain the best materials for significant detection. The irradiative effects demonstrate that these materials are competent in performing as beta sensors providing a significant option to the field of radiation sensors.

The work herein has multiple components including modeling, fabrication, and testing that all provide keys to understanding the beta interactions with the organic materials. MCNP modeling was utilized to better understand the beta interaction/collisions with which each atomic component of the organic molecule. The modeling also allowed SRNL researchers to investigate specific organic variants that have different levels of beta collisions. The organic semiconductor used for these devices was synthesized for SRNL by Dr. John Anthony's research group at the University of Kentucky. Photolithography of sample test structures was accomplished at the CNM clean room fabrication facilities at Argonne National Lab (CNM user proposal \#23377; use of CNM was supported by the U.S. DOE, Office of Science, BES Division, under Contract No. DE-AC02-06CH11357). SRNL researchers also designed and fabricated a specialized testing platform to provide for electrical connections to the OTFT samples studied. Final OTFT device preparation and analysis was accomplished at SRNL including the electrical testing and analysis of the device for ion mobility and device stability. A $50 \mathrm{mCi} \mathrm{Sr} / \mathrm{Y}-90$ beta source was used for the beta exposure testing that analyzed by the electrical response of the transistor. 

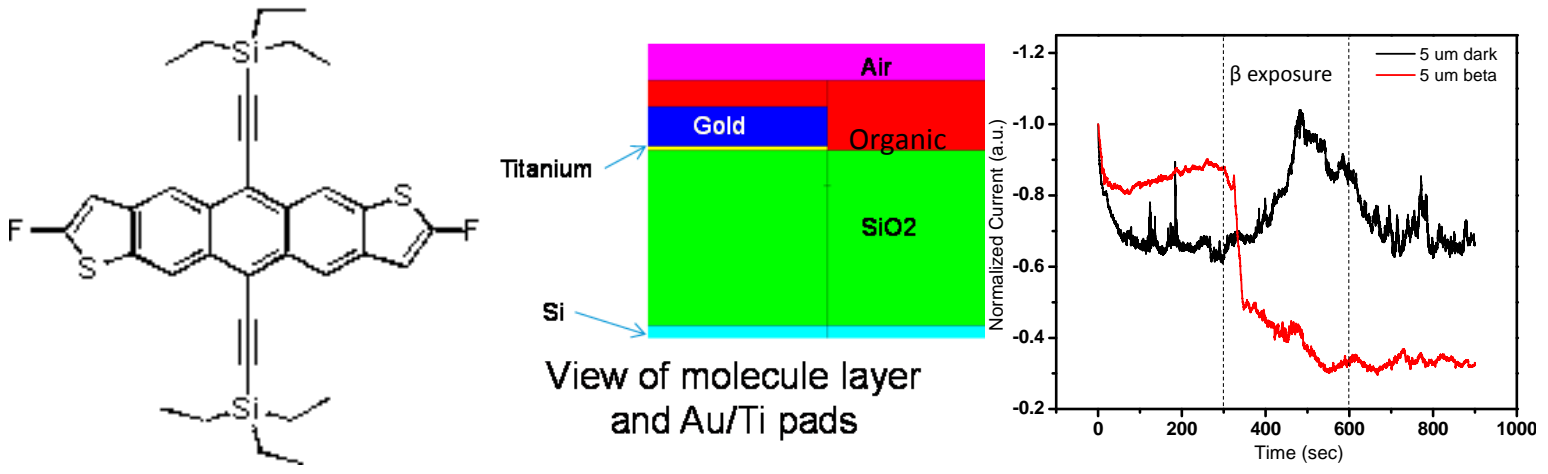

Figure 1. The molecule on the left illustrates the organic molecule utilized in one variant for beta exposure response. The diagram in the middle depicts a structural schematic of the transistor device fabrication by SRNL. The figure on the right illustrates the beta exposure response on the current before and during testing period.

Steady-state electrical measurements showed degradation in the OTFT performance (decrease in source/drain current) over time. A molecular drawing as well as a schematic of the device is shown in figure 1. In addition, the current/time plot shows the device stabilization from the dark the current during the beta exposure. With exposure to beta radiation, an additional decrease in source/drain current was observed and persisted throughout the exposure. The degree of degradation was found to depend on a number of factors including radiation intensity and chemical composition of the organic compound. As an additional analysis for understanding the interactions of beta irradiation on the organic molecule, three chemically similar but separate compounds were irradiated directly as bulk powders. This method of using bulk powders provides a much larger interaction area and a material that can be further analyzed for changes in chemical structure. These samples have been sent back to collaborator at the University of Kentucky for further analysis and processing. Any observed change will be written up and published in collaboration with SRNL. An additional manuscript on the electronic response of OTFTs is in progress and will be completed upon receiving results from collaborator.

Engineering support from the following SRNL colleagues is gratefully acknowledged: R. Lascola, R. Coleman, J. Adamson, and J. Wilson. Additionally, technical support from health physics facility is gratefully acknowleged: I. Brightharp, M. Hollman-Abbott, and J. Barth.

1. Teague, L. C.; Loth, M. A.; Anthony, J. E., Influence of film structure and light on charge trapping and dissipation dynamics in spun-cast organic thin-film transistors measured by scanning Kelvin probe microscopy. Applied Physics Letters 2012, 100 (26), 263305.

2. Binda, M.; Natali, D.; Sampietro, M.; Agostinelli, T.; Beverina, L., Organic based photodetectors Suitability for X- and Gamma-rays sensing application. Nucl. Instrum. Meth. A 2010, 624 (2), 443-448. 
New Materials for Hybrid Lithium-Ion Battery (J.A. Teprovich Jr., H.R. Colón-Mercado, S. Greenway, L. Teague, R. Zidan)

Abstract: Lithium ion batteries are considered the primary energy storage to power electronic devices and transportation vehicles, however deficiencies in current high energy storage capacity and cycle life have hindered their wide spread use. Research on alloy electrodes has shown high energy densities (e.g., 1000-4200 mAh/g) [1]. However their irreversible capacity and capacity fade hindered the advance of the alloy anodes which is caused by large volume changes and irreversible reactions. Herein, we report the testing of a new set of nano-composite anode electrode materials previously developed at SRNL (Basic Energy Science (BES) research program for hydrogen storage) in a Li-ion battery system. Our results show that these materials offer high capacities close to pure metal anodes, however, their irreversible capacities with cycling remain high. Ongoing work is being carried out to extract the kinetic parameters to understand the competing reactions during cycling.

The use of hydrides as electrodes for Li-ion batteries was first demonstrated by Oumellal et al. in 2008 [2]. When using metal hydrides as anode electrodes the following reaction takes place: $\mathrm{MH}_{X}+x \mathrm{Li}^{+}+x e^{-} \rightarrow x \mathrm{LiH}+\mathrm{M}$. The use of hydrides as the negative electrodes for lithium ion batteries represents a new breed of materials with the possibility to alleviate many of the draw backs of carbon and alloy based electrodes. While research in this area is still in its infancy, the successful demonstration of high capacity metal hydrides and semi-reversible cycle testing will have an impact in the lithium ion battery research. The technical objective of this work was to test high capacity, never before used, hydrides as negative electrodes and extract reaction kinetic parameters.

$\mathrm{NaAlH}_{4}$ was first spex-milled for $1 \mathrm{hr}$, while $\mathrm{LiAlH}_{4}$ and $\mathrm{AlH}_{3}$ were used as received. The anode material consisted of the metal hydride, acetylene black, and carboxymethylcellusose in a 1:1:1 weight ratio. The composites were prepared by hand mixing the components in a mortar and pestle. Swagelok type cells were assembled using 7-10mg of the anode composite, Whatman glass fiber filter paper and 1.0M $\mathrm{LiPF}_{6}$ in 1:1 dimethyl carbonate (DMC):ethylene carbonate (EC) as separator/electrolyte, and a $1 \mathrm{~cm}^{2}$ Li foil disk. All cells were assembled/disassembled in an argon filled glovebox. Figure (1) on the right shows the testing of Swagelok type cells. During the constant current charge/discharge the cells were

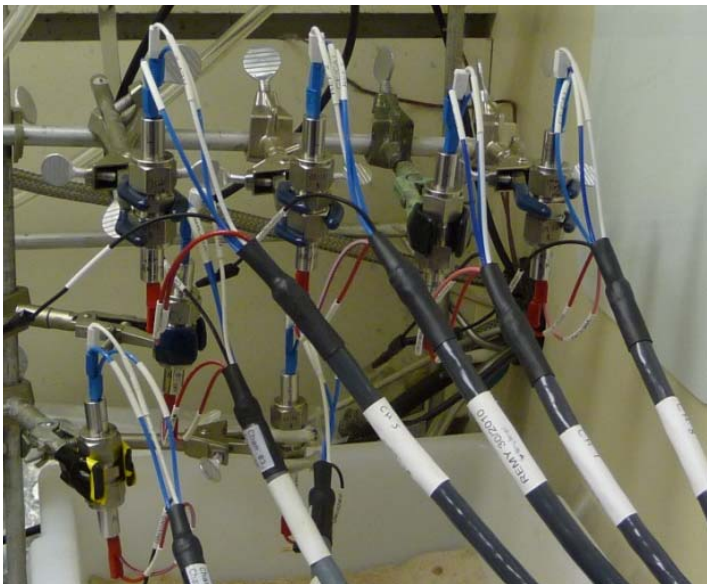

Figure 1. Swagelok type cells used for testing the hydride as anode material. cycled a rate of 1 equivalent lithium in 10 hours between $3.000 \mathrm{~V}$ and $0.005 \mathrm{~V} \mathrm{vs}$. $\mathrm{Li}^{+} / \mathrm{Li}^{0}$. Cyclic voltammograms (CVs) were collected at a cycling rate of $0.1 \mathrm{mV} / \mathrm{s}$ between $3.000 \mathrm{~V}$ and $0.005 \mathrm{~V}$ vs. $\mathrm{Li}^{+} / \mathrm{Li}^{0}$. Electrochemical impedance spectroscopy (EIS) was explored from $50 \mathrm{kHz}$ to $2 \mathrm{mHz}$ under a $10 \mathrm{mV}$ perturbation amplitude at open circuit. 


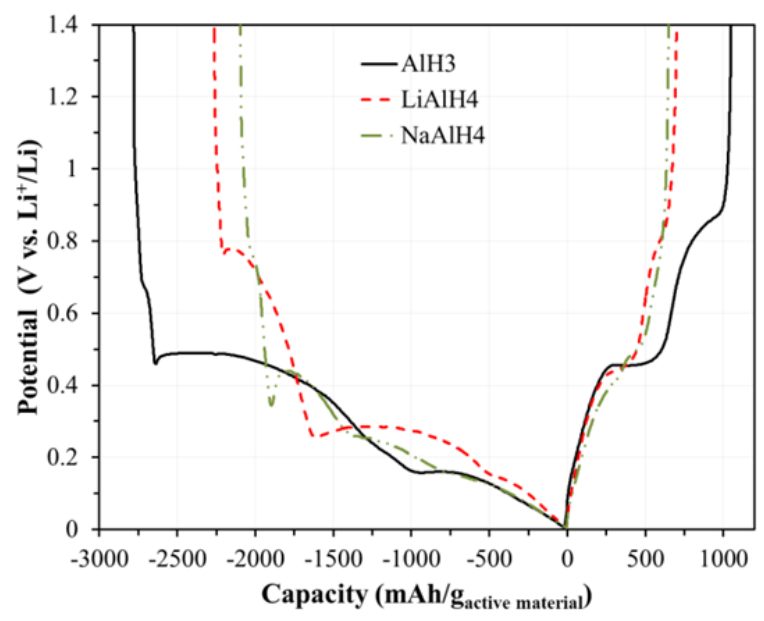

Figure 2. First full discharge/charge capacity. Cycled between $3 \mathrm{~V}$ and $0.005 \mathrm{~V}$.

The first task that was performed was to coordinate a collaborative effort with our no cost partner from the Institut de Chimie et des Matériaux de Paris Est (ICMPE). As the group at ICMPE (led by Dr. Michel Latroche) has prior experience utilizing high capacity metal hydrides as electrodes in lithium ion batteries, this effort allowed us to advance our knowledge of this new system in a very short period.

The figure on the left shows a comparison of the first full charge/discharge cycle (FDC) of $\mathrm{AlH}_{3}, \mathrm{LiAlH}_{4}$ and $\mathrm{NaAlH}_{4}$. The first discharge capacity of the $\mathrm{AlH}_{3}, \mathrm{LiAlH}_{4}$ and $\mathrm{NaAlH}_{4}$ is around $2760 \mathrm{mAh} / \mathrm{g}$ (theoretical 2679 $\mathrm{mAh} / \mathrm{g}$ ), $2260 \mathrm{mAh} / \mathrm{g}$ (theoretical 2118mAh/g) and $2090 \mathrm{mAh} / \mathrm{g}$ (theoretical $1488 \mathrm{mAh} / \mathrm{g}$ ), respectively.

The higher values (than theoretical) are attributed to the irreversible formation of the solid electrolyte interface (SEI). The formation of the SEI is manifested as a small plateau starting at around $0.7 \mathrm{~V}$ during the first discharge cycle. Following the SEI plateau, there are three main plateaus observed: for $\mathrm{AlH}_{3}$ at $0.49 \mathrm{~V}, 0.233 \mathrm{~V}$ and $0.15 \mathrm{~V}$, for $\mathrm{LiAlH}_{3}$ at $0.75 \mathrm{~V}, 0.29 \mathrm{~V}$ and $0.14 \mathrm{~V}$, and for $\mathrm{NaAlH}_{4}$ at $0.42 \mathrm{~V}, 0.24 \mathrm{~V}$ and $0.14 \mathrm{~V}$. These plateaus are believed to be correlated to the reaction of the available hydrogen available to react with the $\mathrm{Li}$ ion to form $\mathrm{LiH}$ and the lithiation of the aluminum. During the charging process, only two main plateaus are observed for all the samples $(0.44 \mathrm{~V}$ and $0.79 \mathrm{~V})$. These plateaus are expected to be the result of the de-lithiation of the $\mathrm{Al}$ and oxidation of some of the lithiated species adsorbed on the surface of the electrode.

The reversibility of the anode catalysts are presented on the figure on the right. This figure shows the large irreversible reaction occurs during cycling. Overall, after four cycles the capacity of the hydrides has decreased to $700 \mathrm{mAh} / \mathrm{g}$ for $\mathrm{AlH}_{3}$ and $500 \mathrm{mAh} / \mathrm{g}$ for $\mathrm{LiAlH}_{4}$ and $\mathrm{NaAlH}_{4}$. Overall the lowest performance is observed for $\mathrm{NaAlH}_{4}$. This was un-expected as the complex hydride was expected to have better reversibility. It is speculated that the sodium ion plays a role in the degradation, by exchanging with the lithium in the electrolyte (forming $\mathrm{NaPF}_{6}$ ).

In conclusion, aluminum base hydrides were tested as electrodes for lithium ion batteries. This LDRD project provided key seed data that allowed the team to request and receive additional funding to develop hydride battery electrodes. Research is continuing to understand and improve these electrodes as part of

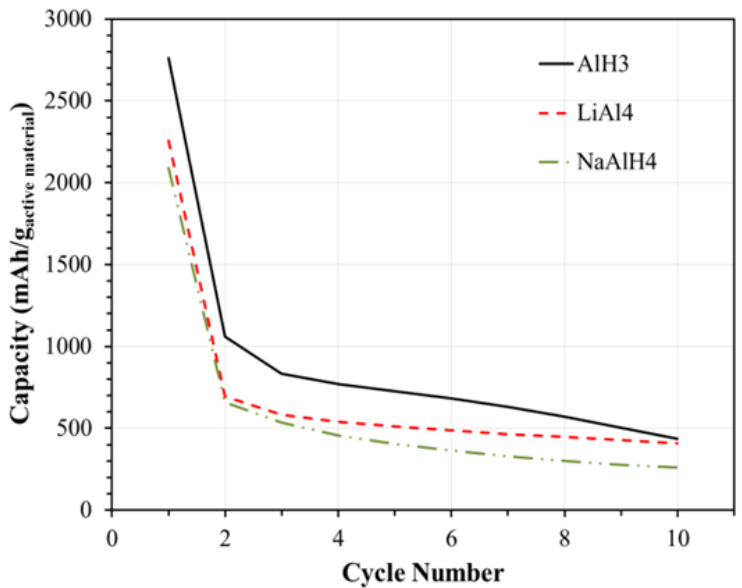

Figure 3. Capacity fade as a function of cycling for the hydride materials. that project funded by the BATT program. That program is a $\$ 450,000$ effort over 2 years with the potential for continued funding if technical milestones are met. 
1. D. Larcher, S. Beattie, M. Morrette, K. Edström, J-C. Jumas and J-M. Tarascom, J. Mater. Chem., 2007, 17, 3759-3772.

2. Y. Oumellal, G. A. Nazri, J-M. Tarascon, L. Aymard, Nature Materials, 2008, 7, 916-921.

\section{Novel Materials for Actinide Separations (K. M. L. Taylor-Pashow (SRNL); W. Lin (UNC); C. W. Abney (UNC); M. Carboni (UNC))}

Abstract: This project aims to develop new materials capable of separating actinides for a variety of applications related to DOE missions. Target applications include treatment of high level nuclear waste $(H L W)$, treatment of acid mine drainage, and extraction of uranium from seawater. Several different types of materials were prepared and evaluated, including functionalized mesoporous carbon and metalorganic framework (MOF) templated materials. Some of the mesoporous carbon materials showed promise for $U$ extraction applications, while the MOF templated materials showed promising strontium and actinide separation capability in a HLW simulant.

There is a need for the separation or extraction of actinide elements in many fields relevant to the DOE mission, for example, nuclear waste processing, environmental clean-up, and development of sustainable nuclear fuel cycles. The objective of this research project was to develop new materials with high selectivity for the separation of actinides under a variety of conditions. Target applications included treatment of high level nuclear waste (HLW), treatment of acid mine drainage, and extraction of uranium from seawater.

Several types of materials were synthesized and examined for actinide separation using simulant solutions. A group of mesoporous carbon materials functionalized with a variety of ligands were developed and evaluated for actinide separations in a HLW simulant, simulated acid mine drainage, and simulated seawater. In addition a new class of nanocomposite materials was synthesized using metalorganic frameworks (MOFs) as templates. These materials were evaluated for strontium and actinide removal from a HLW simulant.

The mesoporous carbon materials were prepared through functionalization of commercially available mesoporous carbon (Aldrich). The mesoporous carbon was functionalized either through in situ formation of a diazonium or through direct oxidation or phosphorylation of the carbon using previously reported methods. ${ }^{1}$ Functional groups included amidoximes, phosphate, phosphonates, and carboxylic acids.

The metal-organic framework template nanocomposites were prepared by synthesis of either an $\mathrm{Fe}^{3+}$ or $\mathrm{Al}^{3+}$ containing metal organic framework of the MIL-101 or MIL-53 structure. ${ }^{2}$ These MOF particles were then coated with a thin layer of $\mathrm{TiO}_{2}{ }^{3}$ Calcination of the sample leads to a mixed oxide nanocomposite with an $\mathrm{Fe}_{2} \mathrm{O}_{3}$ or $\mathrm{Al}_{2} \mathrm{O}_{3}$ core and a $\mathrm{TiO}_{2}$ shell. Some samples were also subjected to treatment with strong base to convert the $\mathrm{TiO}_{2}$ shell to a titanate material. In addition, the uncalcined/TiO 2 coated MOFs, and uncoated MOFs were evaluated, and an amorphous Ti containing coordination polymer was also prepared and evaluated. 
Screening experiments with the functionalized mesoporous carbon materials in the HLW simulant revealed poor performance under these conditions. However, several of these materials showed high affinity for $U$ under acidic $(\mathrm{pH} 3)$ and slightly basic $(\mathrm{pH} 8)$ conditions. Based on these results, the materials were tested in simulated seawater and simulated acid mine drainage solutions. In the simulated seawater solution, the phosphorylated mesoprous carbon sample showed quantitative removal of $U$ at a phase ratio of $0.1 \mathrm{mg} / \mathrm{mL}$ (starting $U$ concentration of $5 \mathrm{ppm}$ ). In the simulated acid mine drainage, no $U$ removal was observed for any of the five materials tested. This is likely due to the $\mathrm{U}$ being present as uranyl sulfate $\left(\mathrm{UO}_{2}\left(\mathrm{SO}_{4}\right)_{3}{ }^{4-}\right)$ in this high sulfate simulant.

Results of sorption testing with the MOF-templated nanocomposite materials in the HLW simulant showed excellent results for $\mathrm{Sr}$ and actinide removal. The uncoated Fe based MOF materials were found to remove $>98 \%$ of the $\mathrm{Sr}$ when added at a total solids concentration of $2 \mathrm{~g} / \mathrm{L}$. This concentration of sorbent provides an Fe concentration of $\sim 0.4 \mathrm{~g} / \mathrm{L}$, which is a factor of 5.5 lower than the $\mathrm{Fe}$ concentration used in previous work by Argonne National Lab for removal of $\mathrm{Sr}$ and actinides using in situ formed mixed iron oxides (IS-MIO). ${ }^{4}$ The uncoated MOF also removed some $\mathrm{Pu}$ and $\mathrm{Np}$ ( 35\%) under these conditions. The $\mathrm{TiO}_{2}$ coating increased both the $\mathrm{Sr}$ and actinide removal performance of the material. When added at an equal concentration $(2 \mathrm{~g} / \mathrm{L})$, the $\mathrm{Sr}$ removal increased to $>99 \%$, the $\mathrm{Pu}$ removal increased to $\sim 80 \%$, and the $\mathrm{Np}$ removal to $\sim 67 \%$. Increasing the concentration of the $\mathrm{TiO}_{2}$ coated Fe MOF materials to $10.4 \mathrm{~g} / \mathrm{L}$ (Fe concentration equivalent to IS-MIO work) resulted in excellent $\mathrm{Sr}$ and actinide removal. Under these conditions $>99.4 \%$ of the $\mathrm{Sr}$ had been removed, along with $94.4 \%$ of the $\mathrm{Pu}, 86.2 \%$ of the $\mathrm{Np}$, and $60.0 \%$ of the $\mathrm{U}$ after 24 hours of contact. Replacing the Fe MOF in the core of this material with an AI MOF increased the actinide removal percentages. Treatment of the HLW simulant with $10.4 \mathrm{~g} / \mathrm{L}$ of the $\mathrm{TiO}_{2}$ coated Al MOF for 24 hours resulted in removal of $>99.7 \%$ of the $\mathrm{Sr}$, $99.4 \%$ of the $\mathrm{Pu},>\mathbf{8 6 . 5 \%}$ of the $\mathrm{Np}$, and $92.9 \%$ of the $\mathrm{U}$. It is expected that the MOF will decompose upon exposure to the highly alkaline HLW simulant forming Fe or Al oxides/hydroxides. Interestingly, the amorphous $\mathrm{Ti}$ containing coordination polymer was found to have comparable $\mathrm{Sr}$ removal performance to the $\mathrm{TiO}_{2}$ coated Fe MOF, but much greater actinide removal performance.

The calcined mixed oxide materials (i.e. $\mathrm{Fe}_{2} \mathrm{O}_{3}$ coated with $\mathrm{TiO}_{2}$ and $\mathrm{Al}_{2} \mathrm{O}_{3}$ coated with $\mathrm{TiO}_{2}$ ) also performed well in the HLW simulant. To compare results to the baseline material, monosodium titanate (MST), these materials were tested at a concentration of $1.6 \mathrm{~g} / \mathrm{L}$, which provides an equivalent $\mathrm{Ti}$ concentration to $0.4 \mathrm{~g} / \mathrm{L} \mathrm{MST}$ (the current baseline). Compared to MST, the untreated $\mathrm{Fe}_{2} \mathrm{O}_{3}$ coated with $\mathrm{TiO}_{2}$ was found to have lower DFs for $\mathrm{Sr}, \mathrm{Pu}$, and $\mathrm{U}$, and a similar DF for $\mathrm{Np}$. However, after treatment of these materials with $10 \mathrm{M} \mathrm{NaOH}$ or $\mathrm{KOH}$, the $\mathrm{Sr}$ removal performance improved greatly, while the actinide removal decreased or remained similar to the untreated samples. Replacing the $\mathrm{Fe}_{2} \mathrm{O}_{3}$ core with $\mathrm{Al}_{2} \mathrm{O}_{3}$ resulted in an increase in performance compared to the untreated $\mathrm{Fe}_{2} \mathrm{O}_{3}$ analog. This material had comparable DF values with the baseline MST.

In conclusion, several new materials have been developed and evaluated for actinide removal under various conditions. A few promising candidates have been identified and warrant additional testing and optimization. Phosphorylated mesoporous carbon was found to quantitatively remove $U$ from a simulated seawater solution under the conditions tested. Additional testing is needed to optimize the loading and elution parameters for this material. Several MOF templated materials were found to show 
excellent strontium and actinide removal performance from a simulated HLW solution. Demonstrating removal performance under these harsh conditions of extreme $\mathrm{pH}$ is a significant achievement for these materials, opening the door to a new area of application for metal-organic frameworks.

1. (a) Li, Z.; Yan, W.; and Dai, S., Langmuir, 2005, 21, 11999-12006. (b) Mayes, R. T.; Fulvio, P. F.; Ma, Z.; and Dai, S., Phys. Chem. Chem. Phys. 2011, 13, 2492-2494. (c) Stein, A.; Wang, Z.; and Fierke, M. A., Adv. Mater. 2009, 21, 265-293.

2. (a) Férey, G.; Mellot-Draznieks, C.; Serre, C.; Millange, F.; Dutour, J.; Surblé. S.; and Margiolaki, I., Science, 2005, 309, 2040-2042. (b) Serre, C.; Millange, F.; Thouvenot, C.; Noguès, M.; Marsolier, G.; Louër, D.; and Férey, G., J. Am. Chem. Soc. 2002, 124, 13519-13526.

3. deKrafft, K. E; Wang, C.; and Lin. W., Adv. Mater. 2012, 24, 2014-2018.

4. Arafat, H. A.; Aase, S. B.; Bakel, A. J.; Bowers, D. L.; Gelis, A. V.; Regalbuto, M. C.; and Vandergrift, G. F., AlChE Journal, 2010, 56, 3012-3020.

\section{Removal of Aqueous ${ }^{99} \mathrm{Tc},{ }^{129} \mathrm{I}$, and ${ }^{137} \mathrm{Cs}$ with Reactive, Low-Cost Materials (D. I. Kaplan, A. S. Knox, K. P. Crapse, D. Li, D. P. DiPrete)}

Abstract: Technetium-99 and ${ }^{129} \mathrm{I}$ are two of the three (along with ${ }^{14} \mathrm{C}$ ) most common risk drivers in low-level and high-level waste disposal sites and among the most common environmental contaminants at DOE sites. ${ }^{137} \mathrm{Cs}$ is the most common contaminant after nuclear accidents, such as Chernobyl or Fukushima, and a key radionuclide-of-concern from weapons of mass effect (WMEs). The objective of this project was to test highly reactive and low-cost materials for removal of these three contaminants from the aqueous phase. Twenty-five sorbents were screened, of which ten were found to be highly effective for removal of aqueous ${ }^{137} \mathrm{Cs}\left(\mathrm{K}_{d}=11,900 \mathrm{~mL} / \mathrm{g}\right),{ }^{129} \mathrm{I}\left(\mathrm{K}_{\mathrm{d}}=29,000\right.$ $\mathrm{mL} / \mathrm{g})$, and/or ${ }^{99} \mathrm{Tc}\left(\mathrm{K}_{\mathrm{d}}=>234,000 \mathrm{~mL} / \mathrm{g}\right)$ (Table 1). The down-selected sorbents included three organoclays (Figure 1; for Cs, I, and Tc), illite (for Cs), argentite (AgS; for I), apatite/fish bones (for I), and chitosan (for Cs, I, and TC). Key parameters were measured to identify the range of appropriate geochemical conditions and to facilitate the design of engineered solutions for applications for environmental cleanup, nuclear waste separation, and WMEs. These results will provide the applied scientific foundation for solving critical DOE and industrial issues in a sustainable and cost-effective manner. The multidisciplinary team submitted a patent disclosure related to two of the highly effective, low-cost organoclays for the sorption of Tc and I.

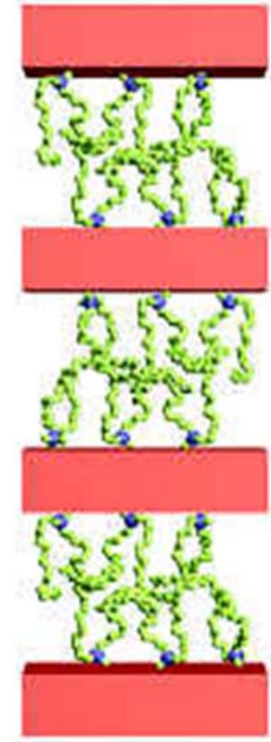

Figure 1. Organo-clays are mined clays amended with surface modified organic compounds designed to enhance contaminant sorption.

Table 1. Cesium, iodine (as iodide, $\mathrm{I}^{-}$) and technetium (as pertechnetate, $\mathrm{TcO}_{4}{ }^{-}$) distribution coefficients $\left(\mathrm{K}_{\mathrm{d}}=(\mathrm{Ci} / \mathrm{g}) /(\mathrm{Ci} / \mathrm{mL})=\mathrm{mL} / \mathrm{g}\right)$ in SRS groundwater ( 2 to 4 replicates).

\begin{tabular}{|c|c|c|c|c|}
\hline Sorbent & Cs $K_{d}$ & $I K_{d}$ & Tc $K_{d}$ & Possible sorption processes \\
\hline Organoclay-OCM & $\begin{array}{c}1,231 \pm \\
111\end{array}$ & $\begin{array}{c}29,313 \pm \\
401\end{array}$ & $\begin{array}{c}>112,229 \pm \\
1442\end{array}$ & $\begin{array}{l}\text { Tc - reductive precipitation via sulfides; } \\
\text { anion exchange, I - anion exchange, } \\
\text { organo-I covalent bonding; Cs - cation }\end{array}$ \\
\hline
\end{tabular}




\begin{tabular}{|c|c|c|c|c|}
\hline & & & & exchange \\
\hline Organoclay OCB & $\begin{array}{c}2,803 \pm \\
572\end{array}$ & $\begin{array}{c}9,613 \pm \\
626\end{array}$ & $\begin{array}{l}>116,798 \pm \\
6823\end{array}$ & $\begin{array}{l}\text { Tc - anion exchange; I- anion exchange, } \\
\text { organo-I covalent bonding; Cs - cation } \\
\text { exchange }\end{array}$ \\
\hline $\begin{array}{l}\text { Surfactant } \\
\text { Modified } \\
\text { Chabazite }\end{array}$ & $>6,591$ & $\begin{array}{c}122 \pm \\
13\end{array}$ & $\begin{array}{c}25,563 \pm \\
1,128\end{array}$ & $\begin{array}{l}\text { Tc - anion exchange, reductive } \\
\text { precipitation as } \mathrm{TcO}_{2}, \mathrm{TcO}_{4}^{-} \text {fitting into } \\
\text { mineral pores; } \mathrm{Cs} \text { - cation exchange }\end{array}$ \\
\hline Illite (IMt-2) & $\begin{array}{c}11,700 \pm \\
3,900\end{array}$ & $\begin{array}{c}\text { Not } \\
\text { measured }\end{array}$ & $\begin{array}{c}\text { Not } \\
\text { measured }\end{array}$ & $\begin{array}{l}\text { Cs - Sterically and near-irreversibly } \\
\text { bound to certain interlayers of mineral }\end{array}$ \\
\hline $\begin{array}{l}\text { Modified Y- } \\
\text { Zeolite }\end{array}$ & $\begin{array}{c}\text { Not } \\
\text { measured }\end{array}$ & $\begin{array}{c}\text { Not } \\
\text { measured }\end{array}$ & $\begin{array}{l}>234,459 \pm \\
1128\end{array}$ & $\begin{array}{l}\text { Anion exchange; } \mathrm{TcO}_{4}^{-} \text {fit into mineral } \\
\text { pores }\end{array}$ \\
\hline Argentite (AgS) & $\begin{array}{c}\text { Not } \\
\text { measured }\end{array}$ & $>25,000$ & $\begin{array}{c}\text { Not } \\
\text { measured }\end{array}$ & Precipitation of AgI \\
\hline SRS sediment & $10-50$ & $0.3-0.9$ & $0.6-1.8$ & $\begin{array}{l}\text { Cs, Cs, and I - Surface complexation with } \\
\text { iron oxides and anion/cation exchange. }\end{array}$ \\
\hline
\end{tabular}

\section{Evaluation of Hydrogen Isotope Exchange Methodology on Adsorbents for Tritium Removal (G. A. Morgan, Jr.)}

Abstract: We have demonstrated a potential process that can be used to remove tritium from contaminated water using Pt-catalyzed molecular sieves. The process is an elemental isotope exchange process in which $\mathrm{H}_{2}$ (when flowed through the molecular sieves) will exchange with the adsorbed water, $\mathrm{D}_{2} \mathrm{O}$, leaving $\mathrm{H}_{2} \mathrm{O}$ absorbed on the molecular sieves. Various formulations of catalyzed molecular sieve material were prepared using two different techniques, Pt-implatation and Pt-ion exchange. This technology has been demonstrated for $a \mathrm{H}$ and $\mathrm{D}$ system, but can also be used for the removal of tritium from contaminated water $\left(\mathrm{T}_{2} \mathrm{O}, \mathrm{HTO}\right.$, and DTO) using $\mathrm{D}_{2}$ (or $\left.\mathrm{H}_{2}\right)$.

The primary technical objective of this research activity was to identify and experimentally verify materials that exhibit good hydrogen isotope exchange activity, high water absorption capacity with minimum tritium retention, and good thermostability. The research activity addressed (and subsequently) answered the following questions:

1. Identification of potential candidate materials for elemental hydrogen isotope exchange;

2. The catalyst level that is needed to facilitate the elemental exchange;

3. How much exchange gas is needed to reduce the inventory of the heavy isotope in the adsorbent.

Tritium contaminated water is a critical issue for nuclear power reactors, particularly when ground water is contaminated by leakage. A case on hand is Vermont Yankee Nuclear PowerStation. It was discovered last year that the tritium level in a ground water monitor well reached 293,000 pCi/liter vs. NRC's required level to report of $30,000 \mathrm{pCi} /$ liter. ${ }^{1,2}$ Planned tritium plants for ITER and LIFE will be required to process huge volumes of tritium contaminated water. ${ }^{3}$ The most practical process for removing the tritiated water vapor from the non-catalyzed molecular sieve beds currently involves heating the bed and passing the desorbed gas through a hot magnesium bed, which converts the water vapor into $\mathrm{MgO}$ and hydrogen $\left(\mathrm{H}_{2}, \mathrm{D}_{2}\right.$, or $\left.\mathrm{T}_{2}\right)$. When the $\mathrm{Mg}$ is completely consumed, the $\mathrm{Mg}$ bed is removed from the process and disposed of as low level radioactive waste. This project has successfully 
demonstrated that $\mathrm{H}_{2}$ will exchange with the $\mathrm{D}_{2}$ present in the $\mathrm{D}_{2} \mathrm{O}$. Even though additional development and testing is necessary (using $\mathrm{T}_{2} \mathrm{O}$ and $\mathrm{D}_{2}$ ), there is definite potential that this technique could be used in facilities that process tritiated water. This would result in the generation of less radioactive waste, resulting in reduced operating costs. In addition, this process is expected to offer better efficiency than the current $\mathrm{Mg}$ bed technology. This new process has the potential to establish SRNL's leadership in handling tritium contaminated water and strengthen the SRNL/SRS leadership in overall tritium processing technology that includes tritium separation, purification, storage, confinement, accountability and monitoring.

There were two phases of this research project. The first phase of the project was to identify potential candidate materials for the elemental hydrogen exchange and to prepare Pt-catalyzed molecular sieve materials. Based on the comparison of the key selection criteria, it was determined that the preferred material that would exhibit hydrogen isotope exchange properties is a Pt-catalyzed Y-type zeolite. The Pt-catalyzed molecular sieve materials were prepared using two different methods. The first method was a Pt-impregnation method where the Pt was deposited directly on the surface of the molecular sieves (commercially available Y-type zeolite). In the second method, the Pt was ion exchanged with the hydrogen ions in the molecular sieve framework and becomes part of the structure of the molecular sieve. Various formulations (at $1.0 \%$ and $1.5 \%$ Pt loading) using the two methods were prepared and subsequently tested for hydrogen isotope exchange properties. The Pt-catalyzed molecular sieve materials were loaded in the experimental test cells and subsequently dried to remove any residual moisture absorbed on the molecular sieves. Once dried, the Pt-catalyzed molecular sieve material was loaded with $\mathrm{D}_{2} \mathrm{O}$. Protium, $\mathrm{H}_{2}$, was flowed through the test cell under ambient conditions where the $\mathrm{H}_{2}$ exchanges with the $D_{2}$ in the $D_{2} \mathrm{O}$ leaving $\mathrm{H}_{2} \mathrm{O}$ absorbed on the molecular sieves. Residual gas analysis was used to verify the hydrogen isotope exchange and determine the efficiency.

All 4 of the Pt-catalyzed molecular sieve samples (1.0\% and $1.5 \%$ Pt deposited on the surface of the molecular sieves and $1.0 \%$ and $1.5 \%$ Pt ion exchanged) showed clear evidence of hydrogen isotope exchange activity. In each of the experimental tests, the hydrogen (protium) exchanges with the deuterium in the $\mathrm{D}_{2} \mathrm{O}$ absorbed on the molecular sieves. In the figures below, there are increases in the mass spectrometer signals for masses 3 and 4 ( $H D$ and $D_{2}$, respectively) and a marked decrease in mass $2\left(\mathrm{H}_{2}\right)$. As the $\mathrm{H}_{2}$ gas is flowed through the Pt-catalyzed molecular sieves, the mass 4 signal decreases over time indicating the exchange of $D_{2}$ with $\mathrm{H}_{2}$ in the absorbed $\mathrm{D}_{2} \mathrm{O}$. 

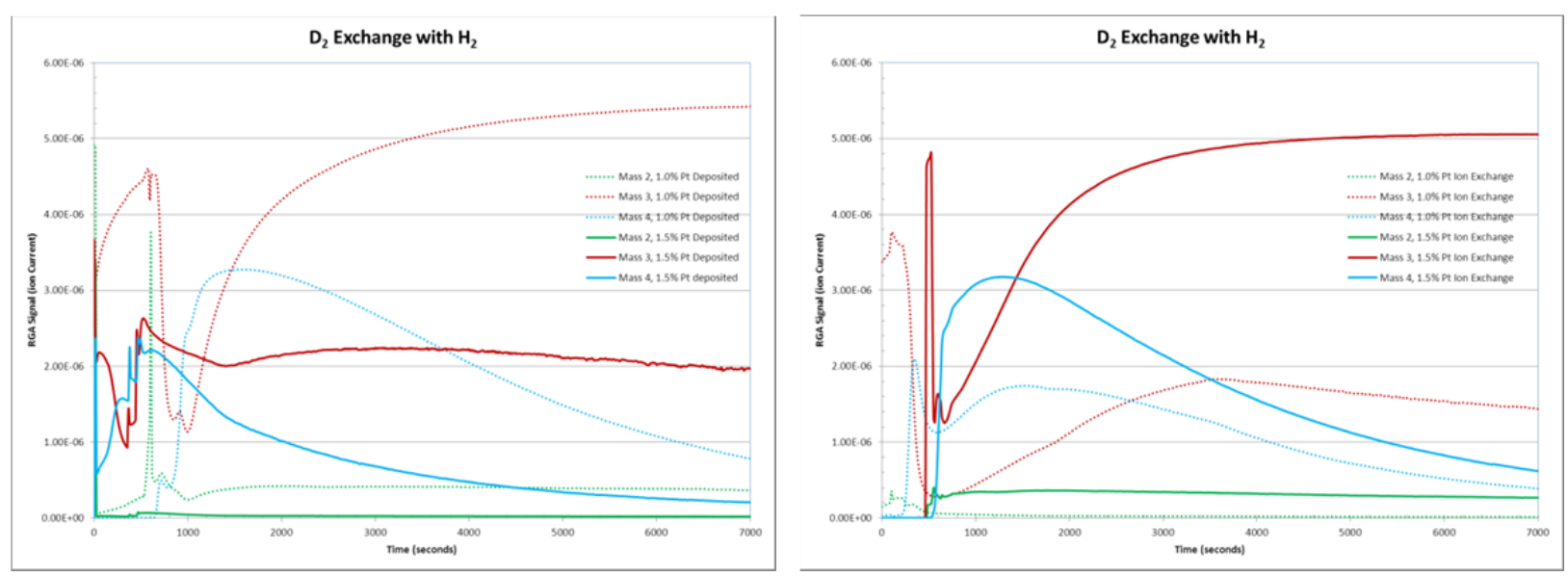

In this LDRD project we have successfully developed and experimentally verified two methods for Ptcatalyzing the molecular sieve material. The first method involves depositing the Pt on the surface of the molecular sieves and the second method is an ion exchange method in which the Pt ions are exchanged with the hydrogen ions in the molecular sieve thus becoming part of the structure. The Ptcatalyzed molecular sieves show hydrogen isotope exchange activity for the 4 samples tested. The molecular sieve samples with the $1.5 \% \mathrm{Pt}$ exhibited the most efficient hydrogen isotope exchange, based on the length of time for the mass 4 signal to decrease.

1. G. Jones, "Tritium Issues in Commercial Pressurized Water Reactors." Fusion Science and Technology, 54, 329, (2008).

2. www.healthvermont.gov

3. D. Murdoch, S. Beloglazov, P. Boucquey, H. Chung, M. Glugla, T. Hayashi, A. Perevezentsev, K. Sessions, and C. Taylor, "ITER Design Review; Tritium Issues." Fusion Science and Technology, 54, 3, (2008).

\section{Application of Cooling Towers for the Collection, Concentration, and Detection of Biological Agents, Radionuclides, and Elemental Chemicals (R. L. Brigmon, D. I. Kaplan, W. L. Jones, M. T. Kingsley (Savannah River National Laboratory, Aiken, SC); S. Leskinen, E. Kearns, D. Lim, (University of South Florida (USF), Tampa))}

Abstract: Cooling towers can serve as constantly-running, passive environmental collectors that can provide suspended particulate, aqueous and biofilm samples for radionuclide and chemical detection. This project demonstrated that further concentration of suspended particulates entrained in the cooling tower water, using the Portable Multi-use Automatic Concentration System (PMACS, a rapid mobile filtration system) lead to increased sensitivity for the detection of solutes, while biofilms also proved to be significant collectors, but were also more variable. Several ion and metal concentrations were greatly enhanced when biofilms and the PMACS was used to sample the cooling towers. PMACS-concentrated cooling tower water samples showed enhanced inductively coupled plasma-mass spectrometry (ICP-MS) detection of cobalt, thorium, uranium, molybdenum, barium, lanthanum, cesium and lead. Isotopic radionuclide analyses at two different facilities provided unique isotopic ratios, consistent with the 
facilities historical use. This validated the use of this monitoring technique for providing signatures (i.e., fingerprints) useful for identifying not only the presence, but the source of the radionuclide. Proteomics analysis of the microbial population indicated species capable of metabolizing or bioprecipitation of target radionuclides and metals, including uranium, from alkaline solutions such as cooling tower water. The project results demonstrate that cooling towers can be exploited as widely dispersed, in situ, chemical and radiological environmental monitoring systems for the signatures and observables of various source terms, including those related to manufacturing or episodic, weapons of mass effect.

The objective of this project was to demonstrate that cooling towers can be exploited to significantly improve atmospheric monitoring. The capacity of cooling towers to collect or entrain compounds from air (1000's $\left.\mathrm{ft}^{3} / \mathrm{min}, 24 / 7\right)$ and the fact that they are ubiquitous world-wide in industrial, municipal, and military environments could prove useful in specific situations. The significance of this approach is the exploitation of existing infrastructure (cooling towers) to serve as environmental collectors to augment traditional environmental monitoring systems including EPA-Radnet (cartridges and precipitation testing). Cooling tower water can be further concentrated quickly and easily using the PMACS, a mobile high-throughput filtration system. Biofilms form in aquatic systems, including cooling towers, when microorganisms attach to mineral or other substrate surfaces and produce films of hydrated extracellular polymers. Reactive functional groups, such as carboxyl, hydroxyl, amino and phosphoryl species, present on biofilm surfaces and the exopolysaccharide matrix, provide a large array of highlyeffective binding sites for metals and radionuclides. In addition, microbial activity may catalyze the transformation of metals and ions into less mobile species as well as provide active uptake of various elements. All of these combined interactions may strongly affect the mechanisms and kinetics of sorption reactions in the aquatic environment of a cooling tower.

Several SRS cooling towers were selected for evaluation of radionuclides and metals detection based on FY11 LDRD research. A total of $30100 \mathrm{~L}$ sample, $31500 \mathrm{ml}$ grab samples, and 22 cooling tower biofilms were collected between January and August 2012. Non-concentrated water samples were taken prior to and immediately after PMACS samples. When present, biofilm samples were collected from cooling tower structures or basins. Due to the variety of tower types at the SRS, those tower basins were sampled repeatedly together with an additional three towers to evaluate the seasonal impact on metal ion targets. Finally, one tower, 285-11F, was sampled repeatedly to examine target compounds over an extended timeframe. In parallel with the water and biofilm testing, one spike and one aerosol spray test using microscopic fluorescent beads was conducted with F-Area Cooling Tower 285-11F in July and August, 2012. PMACS retentates and grab samples were compared for quantities of beads; as well, biofilm samples were examined for the presence/capture of beads. Concentration Ratios (CR) $\left(\mathrm{C}_{\text {solid }} / \mathrm{C}_{\text {aqueous }}\right)$ for ion species were developed for each sample to determine the efficacy of the different sampling methods (retentate vs biofilm) as compared to baseline (water alone). Fluorescent microspheres were introduced to the towers either as an aerosol or directly in the cooling tower basin and then monitored as a function of time to provide a measure of a particles half-life in the cooling tower.

Biofilms were observed to be highly effective at concentrating solutes. Effectiveness: CR for biofilms were: Trivalent Cation > Divalent Cations $>$ Monovalents Cations $=$ Divalent Anions. Biofilms were found 
to be variable within the same cooling tower at sampling times as the biomass in the individual samples varied. The highest concentrations of $U(8.4 \mathrm{ppm}), \mathrm{Cs}(5 \mathrm{ppm})$, Co $(71 \mathrm{ppm})$, and Th $(40 \mathrm{ppm})$ were observed in biofilm samples. The $\mathrm{CR}$ ranged from 700 for $\mathrm{Mn}$, to 35 for $\mathrm{U}$. In many cases target compounds were undetectable in unconcentrated water samples, but analysis of PMACs retentate or biofilms provided enhanced detection. Protein concentrations, an indicator of overall sample biomass, varied from non-detect in water samples up to $290 \mathrm{ug} / \mathrm{ml}$ in retentates. The fluorescent beads persisted for seven weeks in the water and biofilms, indicating the resiliency of particulates in the tower environment. Isotopic radionuclide analyses at two different facilities provided unique isotopic ratios, consistent with the facilities historical use. This validated the use of this monitoring technique for providing signatures (i.e., fingerprints) useful for identifying not only the presence, but the source of the radionuclide. The proteomic analysis results indicated an active biological population in the cooling tower water, despite the application of biocides. Sphingomonas sp. strain BSAR-1 was identified that has been used to bioprecipitate uranium. Acidovorax sp. was also observed that can oxidize iron and uranium when coupled with the reduction of nitrate. A number of petroleum degrading microorganisms were also observed in the analysis. Methylibium petroleiphilum strain PM1 was identified and is one of few isolates that can grow on the fuel additive MTBE (methyl tertiary butyl ether). These bacteria may metabolize ambient diesel/oil fumes and from leaking oil and fuel pumps associated with operations therefore contributing to biofilm growth that enhances capture of metals and radionuclides.

Cooling towers are ubiquitous worldwide in industrial, municipal, and military environments. Knowledge of specific tower operations, e.g water turnover and blowdown, can be used to optimize sampling as successfully shown in this work. The capacity of cooling towers to collect or entrain compounds from air (and retain them for extended periods), as proven here is a novel idea. A patent on this concept has been filed with the US Patent Office. The significance of this approach has garnered attention and FY13 funding from the National Nuclear Security Agency (NNSA). A 2012 manuscript on the work has also been published.

\section{Smart Nanophase Extractors for Environmental Remediation and Liquid Waste Clean-up (L. T. Sexton, R. D. Torres, S. M. Serkiz, B. L. Brown)}

Abstract: Separation and sequestration of long-lived anionic fission products (i.e., ${ }^{99} T c$ and ${ }^{129}$ l) are problematic due to their environmental mobility and redox sensitive speciation (e.g., $\mathrm{Tc}(\mathrm{IV}) \mathrm{O}_{2}$ insoluble versus $\mathrm{Tc}(\mathrm{VII}) \mathrm{O}^{4-}$ aqueous). In this work we fabricated and tested a nanoscale "test-tube and cork" sorbent-based system using the well-developed silica nano-templating method along with silver solid solution and redox chemistries. Experimentally, the interior of the Si-nanotubes were functionalized with silver moieties that are known to be selective for $I$ and $\mathrm{TCO}^{4-}$ removal. Batch sorption studies were then conducted to characterize the sorption behavior of a non-radioactive I isotope to the functionalized nanotubes. We have also shown that after I sorption the nanotubes can be corked with latex particles. The capping efficiency however was found to be effected by the amount of silver present on the template surface. The capped structures could potentially serve as stable waste forms for the long lived anionic fission products. 
Significance/Technical Objective: Separation and sequestration of the long-lived anionic fission products ${ }^{99} \mathrm{Tc}$ and ${ }^{129} \mathrm{I}$ are problematic due to their environmental mobility and redox sensitive speciation. The overall objective of the work described below was to show that hard to sequester anionic fission products (i.e., ${ }^{99} \mathrm{Tc}$ and ${ }^{129} \mathrm{I}$ ) can be separated from solution and sequestered inside capped nano testtubes (i.e., nanophase extractors) for remediation of waste effluents and contaminated groundwater as a stable long-term waste form.

The nanophase extractors to be utilized for the separation and sequestration of Tc and I were successfully prepared using the template synthesis method. This technique involves several steps which include, growth of the nanopourous anodized alumina (AAO) template, sol-gel deposition of silica nano test tubes inside the AAO template, metal functionalization of the silica nano test tubes, and capping of the nano test tubes (performed after sequestration). Figure 1 (inset) shows the SEM image of template embedded Ag doped silica nano test tubes prepared using a 20 wt. \% Ag solution and a chemical filling method. The EDS spectra in Figure 1 confirms that $\mathrm{Ag}$ particles are present on the template, while TEM images (data not shown) of the liberated tubes confirms that the Ag particles are present throughout the length of the nano test tubes.

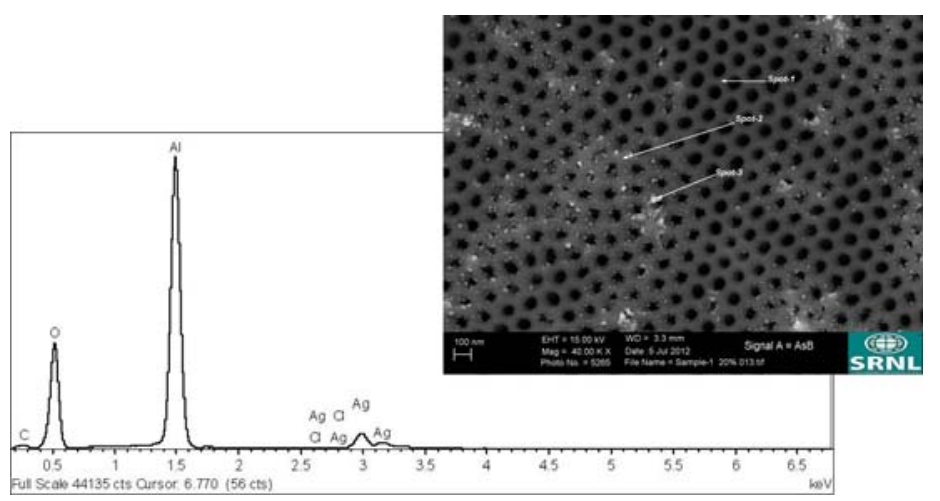

Figure 1. EDS spectra of spot 3 on SEM image (inset) of template embedded Ag doped silica nano test tubes.

Sequestration studies with a non-radioactive isotope of I (i.e., ${ }^{127}$ I) and the template embedded Ag doped silica nano test tubes have been performed. These studies have shown that the $\mathrm{Ag}$ doped tubes do uptake I from solution. The I loading in the silica nano test tubes is dependent on the amount of $\mathrm{Ag}$ inside the tubes. However, the sequestration mechanism is thought to be formation of a Agl(s) surface precipitate:

$$
\mathrm{Ag}(\mathrm{s})+\mathrm{I}^{-}(\mathrm{aq}) \rightarrow \mathrm{Agl}(\mathrm{s})
$$

In sequestration studies performed with a $2 \mathrm{~cm} \times 2 \mathrm{~cm}$ sample of embedded Ag doped silica nanotubes

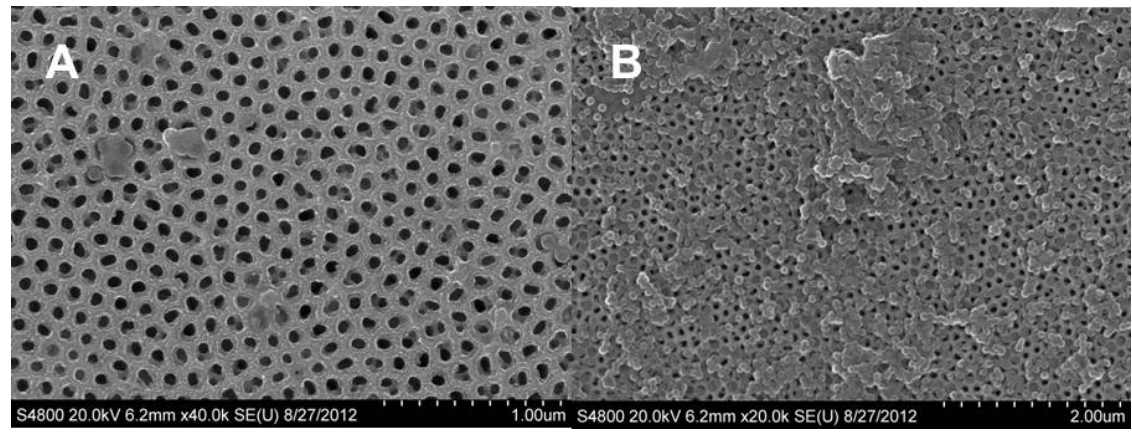
(prepared with a 20 wt. \% Ag solution), the concentration of $\mathrm{I}$ in a $7 \times 10^{-5} \mathrm{M} \mathrm{KI}$ solution dropped by about $13 \%$. A solution containing a control sample (i.e., silica nanotubes with no Ag present) showed no significant change in I concentration. The ${ }^{99} \mathrm{Tc}$ 
sequestration studies were not performed at SRNL during the LDRD period. Samples have been sent to the University of Missouri to perform these studies with both Ag and Fe doped silica nano test tubes.

Following the sequestration studies the template embedded nano test tubes were corked with latex nanoparticles. Capping is achieved by first functionalizing the rim of the tubes with amino silane 3amino-propyltrimethoxysilane (APTS) using silane chemistry. After functionalization with APTS, the nano test-tubes can be spontaneously capped using appropriately sized aldehyde-functionalized latex particles (i.e., Schiff's base reaction to form imine linkages). The capping efficiency was found to be effected by the amount of silver present on the template surface. Figure 2 shows that the capping efficiency of the silica tubes functionalized with a $20 \mathrm{wt}$. \% Ag solution is significantly less than that of those functionalized with a $1 \mathrm{wt}$. $\%$ Ag solution (i.e., $1 \%$ vs. $68 \%$, respectively).

During the course of the LDRD project, the template synthesis method was established at SRNL to create silica nano test tubes to be used as separation and sequestration agents. Utilizing these structures for the separation and sequestration of non-radioactive I was also demonstrated. The studies performed with I indicate that this method could be expanded to the additional species proposed for separation, such as radioactive isotopes of I and ${ }^{99} \mathrm{Tc}$.

Continued work needs to be performed in order to increase the capping efficiency of the Ag doped silica nanotubes. Increased capping efficiency could possibly be achieved by removing the surface layer of $\mathrm{Ag}$ particles found on the template. Removal of the surface layer could be performed by using either a plasma etch or surface swab with nitric acid. Leaching studies of the capped tubes also need to be performed to ensure that the caps prevent the sequestered species from returning into solution. In addition studies with radioactive samples need to be performed. Sorption studies with ${ }^{99} \mathrm{Tc}$ are currently being conducted at the University of Missouri.

Future work in this area could also include identifying additional functional groups/agents to expand the library of nanophase extractors to other long half-life fission products (e.g., ${ }^{90} \mathrm{Sr},{ }^{137} \mathrm{Cs}$ ). Identifying different materials for the nano test tubes could also be another avenue of continued research.

\section{FY 2012 Quick Hit Summaries}

\section{Alignment of Nanorods into Coherent Structures Using Evanescent Electric Fields (R. J. Lascola, P. E. O’Rourke, S. H. Murph, J. S. Wright, K. J. Heroux, B. Peters)}

Abstract: This project investigated the possibility of using a polarized electric field to direct aligned assembly of metal nanorods onto a surface. Aligned nanoparticles (NPs) would allow for cooperative interaction between surface plasmons associated with individual particles, leading to strong optical effects such as enhanced Raman scattering or fluorescence that could be the basis for improved sensors. Nanorods were deposited by evaporation from aqueous suspension on a glass prism while subjected to direct or evanescent laser illumination. We overcame several technical obstacles to create clean NP films and take clear scanning electron microscope images of the films. Despite trying a variety of laser excitation and NP solution conditions and aspect ratios, we did not observe evidence of light-directed 
nanoparticle assembly. We did see evidence of self-assembly that suggests the NP films that were created may, with further development, be the basis for a useful optical chemical sensor.

A plasmon is a coherent dipole oscillation of conduction electrons in a metal. In nanometer-scale metallic nanoparticles (NPs), the resonance frequencies of the oscillations are in the visible and infrared and can be driven by laser excitation. The oscillating electrons generate very strong electric fields close to the surface of the NPs, and any molecules in proximity to the surface will respond to those fields by the production of light by processes such as Raman scattering, fluorescence, or second harmonic generation. Measurement of the light produced in these cases is the basis for a number of chemical sensors with improved detection limits compared to measurements made in the absence of the NPs. Research has shown that the strongest fields occur in the spaces between two adjacent NPs, and it is agreed that the key to reproducibly creating enhanced NP-based sensors is to generate arrays of aligned NPs with a high density of similar interfaces.

The technical objective of this work is to demonstrate that these arrays can be created by using a polarized electric field, e.g. from a laser, to align NPs as they deposit onto a surface. Rod-shaped NPs suspended in a liquid should orient to the electric field in much the same way as a compass needle in a magnetic field. Maintaining the field during the deposition process is necessary to overcome entropic forces during evaporation of the liquid. Once attached to the surface, conventional linker chemistry should be adequate to maintain the aligned orientation. This project explored various aspects of NP solutions, laser powers, and deposition parameters to find the best conditions for generating aligned arrays. Arrays were screened by scanning electron microscopy (SEM).

Two illumination configurations were explored. A $750 \mathrm{~nm}, 10 \mathrm{~mW}$ laser introduced through the dovetails of a quartz prism produced an evanescent field when striking the top surface at a sub-critical angle. This field would only interact with the 1-2 monolayers of NPs closest to the surface. Alternately, top illumination of the prism with a $785 \mathrm{~nm}, 300 \mathrm{~mW}$ laser gave much higher power with a deeper spatial extent. The total illuminated surface area was $\sim 1 \mathrm{~mm}^{2}$. Light was polarized parallel to the prism surface. Gold nanorods $(60 \times 20 \mu \mathrm{m})$, with a resonance at the laser wavelengths, were rinsed, concentrated by centrifugation, and redispersed in water to two concentrations ( $1 x(\sim n M)$ and $10 x)$. 10-20 $\mu \mathrm{L}$ drops were cast onto each prism at the laser-illuminated spot, and allowed to dry either in air, under constant irradiation, or in Ar, with irradiation after the drop dried.

SEM images of the first drops did not show any evidence of NP clustering, due to the presence of a large amount of indeterminate material. Extra cleaning steps (rinsing, centrifugation) removed most of this material and allowed the formation of NP-rich regions on the prism surface. We also determined conditions for obtaining SEM images that avoided damage to the films and prevented charge accumulation (which led to unfocused images).

Ten different combinations of the experimental conditions were investigated. We could not find a set of conditions that produced long-range alignment of NPS. A SEM of the best film is shown below, obtained at high NP concentration, low drop volume, and drying under top-side illumination at high power. Regions of higher (multiple monolayer) and lower (sub-monolayer) NP concentrations are shown at left 
and right, respectively. The higher loadings appear at the edges of the drops. There is some NP alignment but the overall orientation appears random. This suggests that the laser illumination is not driving orientation. At lower coverage, there is less NP alignment. This indicates that cooperative stabilization of multiple NPs may be helpful in overcoming the low energy barrier to NP rotation in solution. (Round particles are aggregated NPs. Aggregate formation during the concentration steps was suppressed in later runs.)
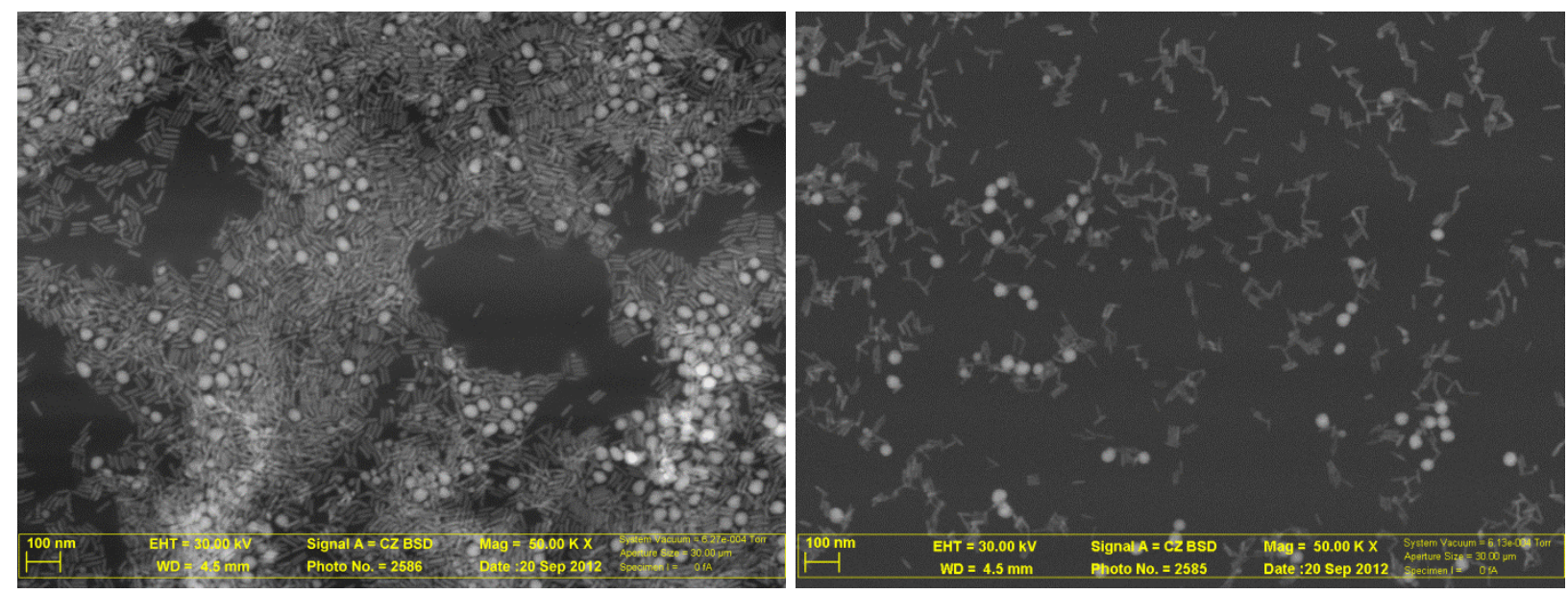

Although we did not reach our goal of creating extensive regions of aligned NPs, this LDRD quick hit project provided some insights towards eventual use of NPs as a sensing platform. Creating regions with higher NP density seems critical to promote alignment and get a higher number of plasmonically active sites. Higher laser powers, and perhaps a more sophisticated alignment, such as counter-propagating beams, may provide enough force to move the particles as they are settling in solution. The apparent self-assembly shown above suggests that films of shaped NPs might have useful properties even if assembly is not aided with external electric fields. Testing of these films, e.g. by measuring a surfaceenhanced Raman scattering effect, would establish baseline levels of reproducibility and sensitivity against which the properties of directed-assembly films could be compared.

\section{Novel Concepts for Isotopic Separation of ${ }^{3} \mathrm{He} /{ }^{4} \mathrm{He}$ (L. E. Roy (PI), H. L. Watson, H. L. Nigg)}

Abstract: The research outlined below established theoretical proof-of-concept using ab initio calculations that ${ }^{3} \mathrm{He}$ can be separated from ${ }^{4} \mathrm{He}$ by taking advantage of weak van der Waals interactions with other higher molecular weight rare gases such as xenon. To the best of our knowledge, this is the only suggested method that exploits the physical differences of the isotopes using a chemical interaction.

The world is experiencing a shortage of ${ }^{3} \mathrm{He}$, a rare isotope of helium with many different critical applications in medical imaging, cryogenics, oil \& gas exploration, scientific research, and in particular, neutron detection. One potential source for ${ }^{3} \mathrm{He}$ outside of using tritium includes extraction of naturally occurring ${ }^{3} \mathrm{He}$ from natural gas. The U.S. Geological Survey estimates that ${ }^{3} \mathrm{He}$ reserves and resources to be between 1 and 5 million liters. To date, there is no practical method to isolate and recover ${ }^{3} \mathrm{He}$ from ${ }^{4} \mathrm{He}$ on a production scale which, if it existed, could take advantage of untapped resources for this vital isotope. ${ }^{3} \mathrm{He}$ constitutes only $1.37 \mathrm{ppm}$ of total $\mathrm{He}$, too dilute to separate economically from ${ }^{4} \mathrm{He}$ (the 
major isotope) by distillation alone. 'Superfluid' separations through a filter are feasible at temperatures of $2.17 \mathrm{~K}$ or less. Membrane separation using porous graphene has been suggested but operating temperatures are predicted to be around $10 \mathrm{~K}$. Despite the information taught in general chemistry textbooks, He will form a weak van der Waals interaction with other rare gases (Rg) such as Xe to form a heteronuclear dimer. Using van der Waals interactions as a separations technique could move the process outside of liquid hydrogen temperatures. The research outlined below used a theoretical approach to explore the weak van der Waals interactions of rare gases (such as Xe) with ${ }^{3} \mathrm{He}$ and ${ }^{4} \mathrm{He}$ to determine if the interaction can be used for He isotope separations.

A series of high-level $a b$ initio calculations at different temperatures for the homonuclear rare gas dimer ${ }^{4} \mathrm{He}_{2}$ (benchmarking) and heteronuclear dimers ${ }^{3} \mathrm{He} /{ }^{4} \mathrm{He}-\mathrm{Xe},{ }^{3} \mathrm{He} /{ }^{4} \mathrm{He}-\mathrm{Ar},{ }^{3} \mathrm{He} /{ }^{4} \mathrm{He}-\mathrm{Xe}-\mathrm{Xe},{ }^{3} \mathrm{He} /{ }^{4} \mathrm{He}-\mathrm{Ar}-\mathrm{Ar}$, ${ }^{3} \mathrm{He}_{2}-\mathrm{Xe}$, and ${ }^{4} \mathrm{He}_{2}-\mathrm{Xe}$ were performed. $\mathrm{He}_{2}$ is the lightest van der Waals system and is difficult to treat accurately because its dissociation energy is small $(11 \mathrm{~K})$ and the attraction is dominated by dispersion forces. The potential energy curves needed for thermodynamic property prediction were created using highly accurate coupled cluster UCCSD-T correlation energy treatments employing correlation consistent basis sets. This provided excellent compromise between accuracy and computational cost to evaluate the concept as a possible separations technique (calculated ${ }^{4} \mathrm{He}_{2}$ dissociation energy is $10.94 \mathrm{~K}$ ). The thermodynamic predictions were then used to estimate partition function ratios at optimized temperatures.

Figure 1 shows the potential energy curve (top) for a single ${ }^{4} \mathrm{He}$ atom bound to an $\mathrm{Ar}$ or Xe atom to create a heterodinuclear molecule. The dissociation energy can be computed from this curve by subtracting the zero point energy from the well depth. The dissociation energy is expressed in wavenumbers and is a measure of the energy required to dissociate the He atom from the rare gas; the larger the number, the stronger the interaction between the atoms. Benchmark calculations show a ${ }^{4} \mathrm{He}-\mathrm{Rg}$ bond dissociation energy of $\sim 22 \mathrm{~cm}^{-1}$ for ${ }^{4} \mathrm{He}-\mathrm{Ar}$ and $\sim 80 \mathrm{~cm}^{-1}$ for ${ }^{4} \mathrm{He}-\mathrm{Xe}$. The higher value for $\mathrm{He}$-Xe indicates a stronger ${ }^{4} \mathrm{He}-\mathrm{Xe}$ attraction than with $\mathrm{Ar}$. The bottom half of Figure 1 shows the formation enthalpy $\left(\Delta \mathrm{H}_{\mathrm{f}}\right)$ as a function of temperature for the binding of ${ }^{3} \mathrm{He} /{ }^{4} \mathrm{He}$ with $\mathrm{Ar}$ and $\mathrm{Xe}$. The graph shows that formation of a ${ }^{3} \mathrm{He}-\mathrm{Xe}$ heterodinuclear molecule is predicted to occur at $\sim 105 \mathrm{~K}$ and for ${ }^{4} \mathrm{He}-\mathrm{Xe}$ at $\sim 108 \mathrm{~K}$. In other words, ab initio calculations predict at $3 \mathrm{~K}$ temperature difference between the two species with the ${ }^{4} \mathrm{He}$ isotope having a stronger interaction to Xe. The partition function ratio for ${ }^{4} \mathrm{HeXe} /{ }^{3} \mathrm{HeXe}$ at $100 \mathrm{~K}$ is 1.015 . This number is the equilibrium constant for the exchange reaction between the separated atoms and the molecule in question. Separation factors can then be calculated from the partition function ratio for the isotopic molecules involved in a reaction. For comparison, the same calculations were performed for the ${ }^{3} \mathrm{He} /{ }^{4} \mathrm{He}-\mathrm{Ar}$ species, but the temperature difference was negligible. The partition function ratio for ${ }^{4} \mathrm{HeAr} /{ }^{3} \mathrm{HeAr}$ at $20 \mathrm{~K}$ is 1.087. 

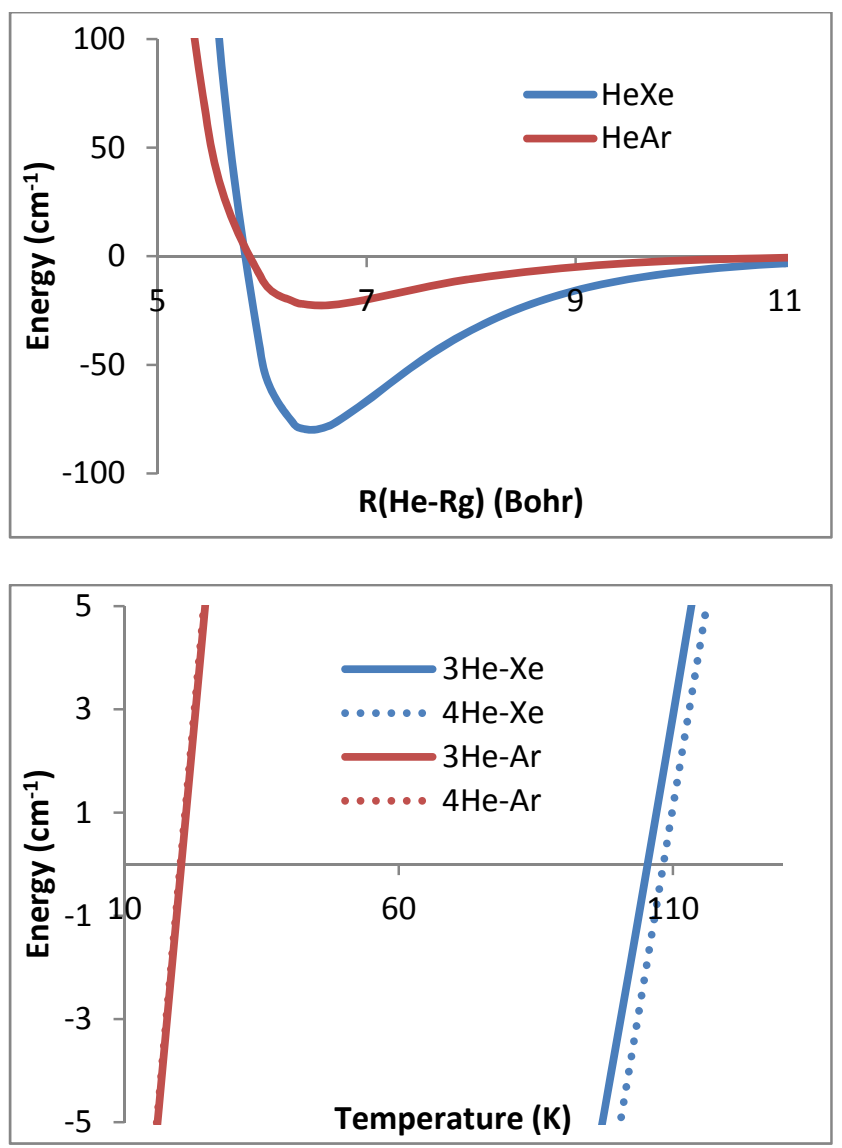

Figure 1. Calculated potential energy surface of ${ }^{4} \mathrm{He}-\mathrm{Rg}(\mathrm{Rg}=$ $\mathrm{Xe}, \mathrm{Ar}$ ) species as a function of distance (Top) and formation enthalpy $\left(\mathrm{H}_{\mathrm{f}}\right.$ ) as a function of temperature (Bottom) for the binding of ${ }^{3} \mathrm{He} /{ }^{4} \mathrm{He}$ with $\mathrm{Ar}$ and $\mathrm{Xe}$ at the UCCSD-T level of theory.

These preliminary data are encouraging and suggest that He isotope separations could occur using van der Waals interactions with Xe. Xe becomes a solid at $161 \mathrm{~K}$ under atmospheric pressure (above the heterodinuclear formation temperature). Further investigation is needed to determine if ideal separation system working conditions would be in the liquid, gas, or supercritical phase. Follow-on funding will be requested to perform additional calculations to improve calculation fidelity, estimate temperature/pressure requirements and to design a benchtop separation system. The benchtop separation system is necessary to demonstrate proof-of-concept of the $a b$ initio results. This may require refined calculation to determine the appropriate operating conditions for the system. Ideally, the system would include a mechanism to separate and recycle Xe. The separation products would be verified using IR and mass spectrometry.

\section{Application of Quantum Cascade Lasers to IR Spectroscopy (T. L. White, P. E. O’Rourke,} W. A. Spencer, F. Fondeur)

Abstract: Infrared absorbance sensors are used extensively to monitor the chemical composition of gas streams for pollution abatement and process control. A primary limitation of these sensors is their 
inability to distinguish a target analyte from an interfering species, especially if the target analyte is present at low levels. Quantum cascade lasers (QCL) light sources can alleviate the interference problem because of their very narrow line widths, continuously tunable wavelength and very high brightness. The high brightness of the QCL should significantly improve light detection and ranging (LIDAR) and infrared (IR) microscopic applications. The development of a hollow core waveguide (HCWG) gas sample cell would take advantage of all QCL properties because gases could be examined at low pressures and long path-lengths, improving sensitivity and resolution, while maintaining a simple and compact sample handling system. In principle, a QCL-HCWG based instrument would be compact and rugged with very high performance characteristics. At SRS, a QCL-IR sensor would have immediate application in tritium processing and hydrogen fuel-cell research for monitoring impurities in hydrogen gas, and environmental monitoring for tracking $\mathrm{CO} / \mathrm{CO} / \mathrm{NOx}$. The sensor would have broad applications throughout the chemical and energy production industries for process control and pollution monitoring.

This work investigated the application of quantum cascade lasers for high resolution infrared spectroscopy of gases and compared their capabilities to FTIR spectroscopy. We chose to use carbon monoxide gas as a baseline analyte because it has strong absorbance in the range of one of our QCL's. The primary features of interest in our experiments are spectral resolution, baseline noise, wavelength

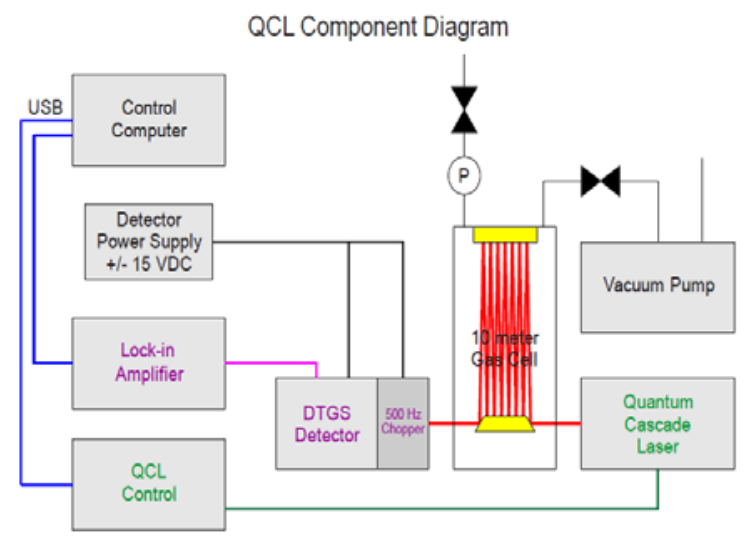
stability, and wavelength reproducibility. We also tested the compatibility of the QCL light source with infrared transmitting hollow core waveguides (HCWG). These HCWG can be used to transmit IR radiation similar to fiber optics for visible light as well as used as very low volume sample cells.

The schematic of the instrument is a layout with a 10 meter gas sample cell in place. The gas cell was evacuated and gas samples were introduced by way of a gas line. Gas samples were examined using the highly collimated QCL beam over a $75 \mathrm{~cm}^{-1}$ range. The beam exiting the sample cell was chopped at $500 \mathrm{hz}$ and then detected using a deuterated triglycine sulfate (DTGS) pyroelectric detector.

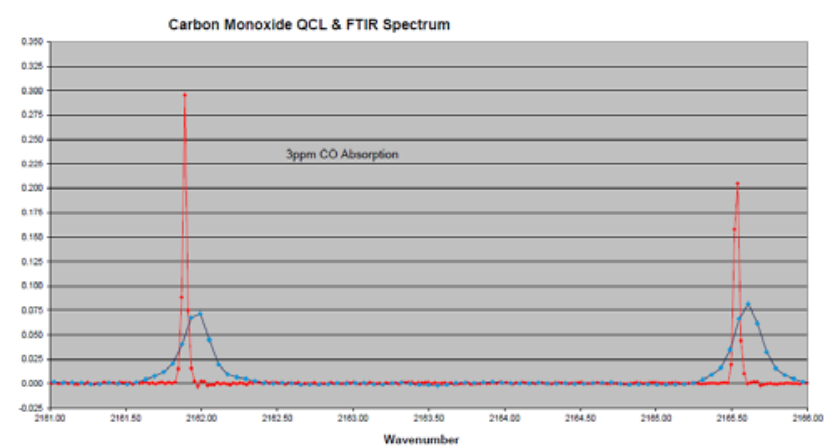
The QCL scan was synchronized using a Lock-in amplifier. This method produce very high resolution spectra $\left(\sim 0.001 \mathrm{~cm}^{-1}\right)$ when compared to typical FTIR as seen in the spectrum of 3ppm CO. Considerable effort was made to synchronize the QCL scan with the lock-in amplifier and aligning $\mathrm{QCL}$ peaks using a custom auto-correlation program.

The use of the QCL system in place of a traditional FTIR produce the advantages of very high resolution, low noise, high power, low beam divergence, and excellent wavelength stability. Further investigation is needed to improve wavelength reproducibility using faster electronic readout and wavelength dithering using current modulation. 


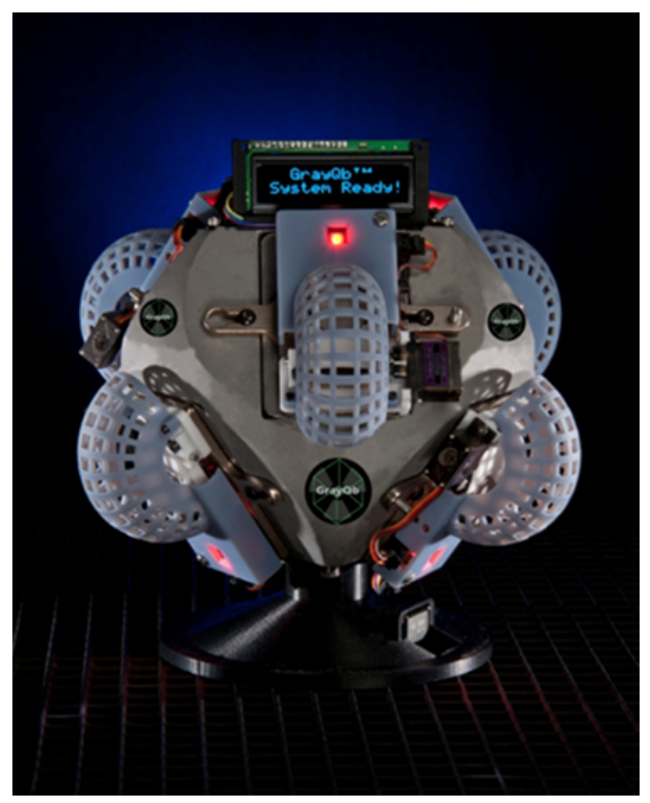

Scientists at the Savannah River National Laboratory have developed and tested a device named GrayQb ${ }^{\mathrm{TM}}$, with accessories, to locate, identify, and generate a map of radioactive contamination within an enclosed area. GrayQb $^{T M}$ is a cubic device that utilizes multiple layers of Phosphor Storage Plates (PSPs) that are highly sensitive, which translates into shorter counting times. Even with low dose rate environments, this device can be used to expedite radioactive contamination cleanup operations. The PSPs from GrayQb ${ }^{T M}$ device are read on a commercially available scanner where special software records and translates the exposure data to define the type and location of the radioactive source(s).

Nuclear facilities that perform cleanup operations as a routine or from an unexpected incident need to determine the location of radioactive contamination in areas such as shielded enclosures or entire laboratory modules. These determinations are typically performed with portable count rate instruments operated by personnel wearing protective gear. These operations can be time-consuming and can expose personnel to unnecessary exposure. Some areas may be too small, confined, or have limited entry for personnel to access. Existing portable instruments may be difficult to deploy in these areas.

The PSPs used for GrayQb ${ }^{\mathrm{TM}}$ are commonly used for imaging purposes where the radiation source and dose rate are known. Common uses are medical imaging and nondestructive testing. This mature technology has been developed with the goal of "minimal dose" in mind, particularly with respect to reducing patient doses from medical imaging. The result is a high resolution, high sensitivity gamma detector material capable of micrometer resolution and $\mathrm{mR}$ levels of dose required. In order to provide energy determination and identification of a radioactive source and its intensity, the PSPs are stacked into a sandwich, separated by attenuation material such as metal, plastic, etc. An unknown radiation source will deposit the most dose to the outer-most PSP, with each successive layer beneath receiving less than the previous one. Based on the amount of exposure to each layer, the energy of the incident radiation can be determined. Modified configurations using other film types can be used for alpha, beta, or neutron detection.

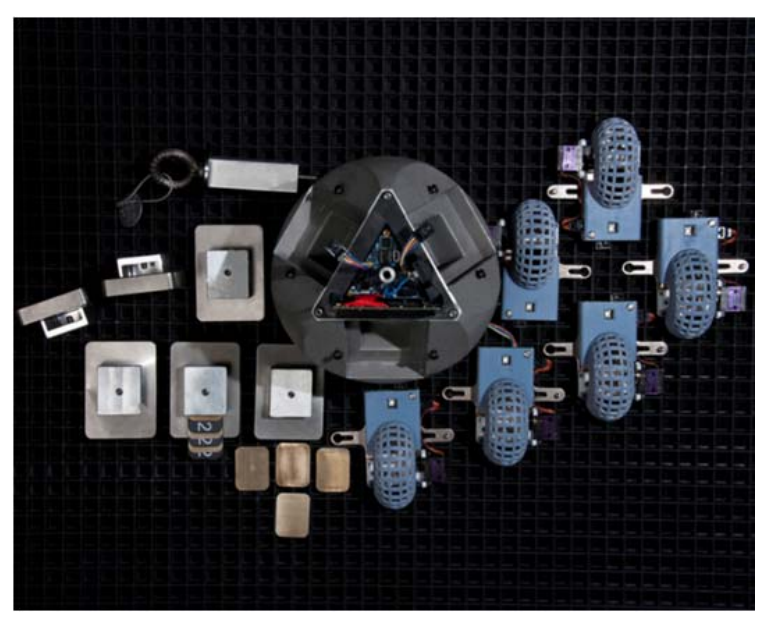


$\mathrm{GrayQb}^{\mathrm{TM}}$ is placed in a confined area for a predetermined span of time based on expected dose rates. A set of motorized shutters shield the PSPs during placement and removal of the device. Once in position, the attenuated PSP layers are exposed to the sources in the area through a unique collimation apparatus. GrayQb ${ }^{\mathrm{TM}}$ is then removed from the area where the PSPs are read in an optical scanner. Using special software, the data from the PSPs is translated into a map of the locations, intensities, and energies of the source contamination.

$\sqrt{ }$ Characterizes contamination at a wide range of dose rates $(0.01$ to over $1000 \mathrm{mGy} / \mathrm{hr} ; 1$ to over100,000 mR/hr)

$\sqrt{\text { Minimizes exposure to personnel }}$

$\sqrt{ }$ On board electronics provides remote control and monitoring of the device

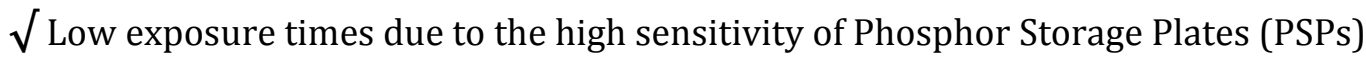

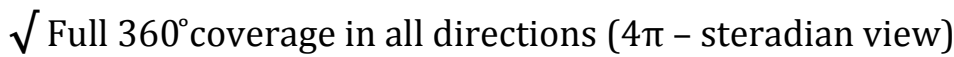

$\sqrt{ }$ Patent pending 


\section{Contract No:}

This document was prepared in conjunction with work accomplished under Contract No. DE-AC09-08SR22470 with the U.S. Department of Energy.

\section{Disclaimer:}

This work was prepared under an agreement with and funded by the U.S. Government. Neither the U. S. Government or its employees, nor any of its contractors, subcontractors or their employees, makes any express or implied: 1. warranty or assumes any legal liability for the accuracy, completeness, or for the use or results of such use of any information, product, or process disclosed; or 2. representation that such use or results of such use would not infringe privately owned rights; or 3 . endorsement or recommendation of any specifically identified commercial product, process, or service. Any views and opinions of authors expressed in this work do not necessarily state or reflect those of the United States Government, or its contractors, or subcontractors. 